PAPER • OPEN ACCESS

\section{Light absorption enhancement and radiation hardening for triple junction solar cell through bioinspired nanostructures}

To cite this article: Thomas Vasileiou et al 2021 Bioinspir. Biomim. 16056010

View the article online for updates and enhancements.
You may also like

- Investigational study on Influence of Fiber
$\frac{\text { Reinforced Polymer Wrapping on }}{\text { Concentrically Loaded Concrete Column }}$
N Pannirselvam, B SudalaiGunaseelan
and J Rajprasad
- Local Thinning Induced Less Oxide
Breakdown in MOS Structures Due to
Lateral Non-Uniformity Effect
Huang-Hsuan Lin and Jenn-Gwo Hwu
- Accuracy limits for the determination of
cortical width and density: the influence of
object size and CT imaging parameters
Sven Prevrhal, Klaus Engelke and Willi A
Kalender

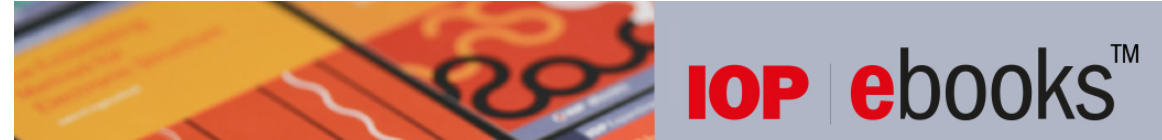

Bringing together innovative digital publishing with leading authors from the global scientific community. Start exploring the collection-download the first chapter of every title for free. 


\section{Bioinspiration \& Biomimetics}

OPEN ACCESS

\begin{tabular}{l} 
CrossMark \\
RECEIIED \\
Rarch 2021 \\
REVISED \\
20 May 2021 \\
ACCEPTED F0R PUBLICATION \\
8 June 2021 \\
PUBLISHED \\
29 July 2021 \\
\hline Original content from \\
this work may be used \\
under the terms of the \\
Creative Commons \\
Attribution 4.0 licence. \\
Any further distribution \\
of this work must \\
maintain attribution to \\
the author(s) and the \\
title of the work, journal \\
citation and DOI. \\
CC \\
BY
\end{tabular}

PAPER

\section{Light absorption enhancement and radiation hardening for triple junction solar cell through bioinspired nanostructures}

\author{
Thomas Vasileiou $^{1, *(D)}$, José M Llorens ${ }^{2}$, Jerónimo Buencuerpo ${ }^{2,3}$, José M Ripalda $^{2}$ (D), \\ ${\operatorname{Dario~} \operatorname{Izzo}^{1}(\mathrm{D}) \text { and Leopold Summerer }}^{1}$ \\ Advanced Concepts Team, European Space Research and Technology Centre (ESTEC), 2201AZ Noordwijk, The Netherlands \\ 2 Instituto de Micro y Nanotecnología, IMN-CNM, CSIC (CEI UAM + CSIC) Isaac Newton, 8, E-28760, Tres Cantos, Madrid, Spain \\ Author to whom any correspondence should be addressed. \\ 3 Present address: National Renewable Energy Laboratory (NREL), 15013 Denver W Pkwy, Golden, CO 80401, United States of America. \\ E-mail: vathomas@zoho.com \\ Keywords: space solar cells, radiation damage, photonic crystals
}

\section{Introduction}

Photovoltaic solar panels are the main power source for Earth-orbiting satellites and have proven their capabilities up to the orbit of Jupiter, with Juno [1] being the solar-powered mission that operated the furthest from the Sun to date. Solar cell efficiency and mass are of great interest for space applications, because improvements to either result in significant reduction in in-orbit solar array costs [2]. Multijunction cells, achieving superior efficiency than their single-junction counterparts, are the norm for powering space vehicles and satellites [3]. However, the high efficiency of the multi-junction cells as produced (beginning-of-life, BOL) is half of the story; the end-of-life (EOL) efficiency has also to be considered, which takes into account the performance degradation during the lifespan of the mission. The space radiation environment constitutes a significant contributor to the aforementioned degradation. Therefore, radiation hardness is an additional and decisive characteristic when selecting solar cells for space operations [4].

The common practice to diminish the radiationinduced effects on solar cells is to place a protective cover glass on their outer surface [5]. The cover glass 'slows down' the spectrum of incoming particles, lessening the damage. A complementary approach is to design the cell to be intrinsically more resilient to radiation. Thinning of the cell junctions facili- 
tates the diffusion driven collection of minority carriers, making the cell less sensitive to the introduction of defects [6-11]. On the downside, thinner junctions absorb less light than their thicker counterparts, which is accompanied by losses on the generated photocurrent. Therefore, the light absorption on thin cells must be enhanced by engineering the optical structure beyond the single pass absorber. Enhanced absorption can be achieved by adding flat mirrors [9, 10] or nanostructures $[12,13]$ in the back of the cell, both of which increase the optical path. However, increasing the absorbed light is more challenging for multi-junction than for single junction cells; the optical structure has to be placed behind the junction which absorbs the least light, otherwise the subsequent junctions-having a lower bandgap - will absorb the incoming photons. This problem has restricted the strategies for compensating the thinning of the middle junction to using distributed Bragg reflectors (DBRs) between the middle and the bottom subcells $[4,8,14]$. These reflective elements are employed in conjunction with a front surface antireflective coating (ARC). Replacing the ARC with a light trapping structure (LTS) that bends the incident light and increase the coupling to the guided modes of the junctions offers the possibility to further compensate for this optical loss [15-22]. Although the current work focuses on space photovoltaics, the described light trapping schemes might also contribute to make III-V materials cost competitive in terrestrial applications by reducing the thickness, and consequently the cost, of III-V solar cells $[12,23,24]$. Similar efforts to reduce the cost of multi-junctions using photonics are currently under way [25].

The problem of maximizing light harvesting or minimizing surface reflectance has been tackled by several living organisms, which have developed elaborate photonic micro- or nanostructures [26, 27]. Specialized nanopore arrays on the transparent hard cell wall of diatoms, a family of single-cell microalga, has been shown to act as a photonic crystal that focuses and confines light [28-31], thus increasing the photosynthetic yield. Furthermore, moths and several butterfly species have evolved an array of protuberances on the cornea of their compound eyes (termed the corneal nipple array), which considerably reduces the reflectance at the surface of their eyes [32-34]. The diatom and the moth-eye structures are well described in the literature and are suitable as alternatives to the widely-used bilayer $\mathrm{ARC}$; therefore, in the following we considered both structures.

The use of a bioinspired nanostructure as a means to improve the light absorption of solar cells has been previously considered, both for single $[35,36]$ and multi-junction solar cells [37-41]. The current paper examines an alternative application for the LTS; as a way to design thin junction solar cells with enhanced radiation hardness, with minimal sacrifice in efficiency. We select a reference triple junction solar cell design that includes a DBR between the middle and bottom junctions [4] and determine the geometry of the bioinspired LTS that achieves the maximum BOL efficiency for a range of middle junction thicknesses. Then, we select the LTS geometry and junction thickness pairs that achieve comparable BOL efficiency in comparison to the reference solar cell and contrast their degradation at various radiation exposures. To this extend, we employed Monte Carlo simulations to quantify the radiation-induced defect density on the semiconductor materials and translate the changes in defect density into efficiency deprecation. Finally, we examine the trade-off between BOL and EOL efficiency.

The paper is organized as follows: in section 2 we present the description and modeling of the solar cell. Specifically, we introduce the solar cell and the LTS geometries, along with their parametrizations in section 2.1. We describe the solar cell model which allows us to estimate the cell efficiency in both the case of a pristine or an irradiated cell in sections 2.2 and 2.3, respectively. The modeling of the radiation damage on semiconductor material is detailed in section 2.4. The optimization procedure that allows us to determine the LTS parameters is outlined in section 2.5. The numerical results are presented in section 3 and further discussed in section 4 . Section 5 presents the conclusions.

\section{Methods}

\subsection{Solar cell and light trapping structures}

We consider a prototypical triple junction space solar cell, based on the Azur 3G30C model [4]. The junctions from top to bottom are manufactured out of GaInP, InGaAs and Ge, as shown in figure $1(\mathrm{a})$, with band gaps at $1.9 \mathrm{eV}, 1.4 \mathrm{eV}$ and $0.66 \mathrm{eV}$. The material compositions were determined by matching the ternary lattice to the Ge substrate and is summarized in table 1. The ARC or the LTS is placed between the solar cell and the protective cover glass. The thickness of the top and middle junctions were determined from [4, figure 7] to be $0.60 \mu \mathrm{m}$ and $1.87 \mu \mathrm{m}$ respectively. In the actual $3 \mathrm{G} 30 \mathrm{C}$ solar cell, a DBR is located in between the middle and bottom junctions. The DBR increases the photon recycling by reflecting the photoluminescence of the middle junction. For the sake of simplicity, we have abstracted the DBR layer by a selective perfect mirror (SPM) characterized by a perfect reflectance at wavelengths shorter than the wavelength that corresponds to the middle junction bandgap and perfect transmittance otherwise. Although the design of the DBR is not trivial, it can be isolated from the design of the LTS.

For the LTS, the underlying absorbing substrates have dissimilar optical properties from the ones found 


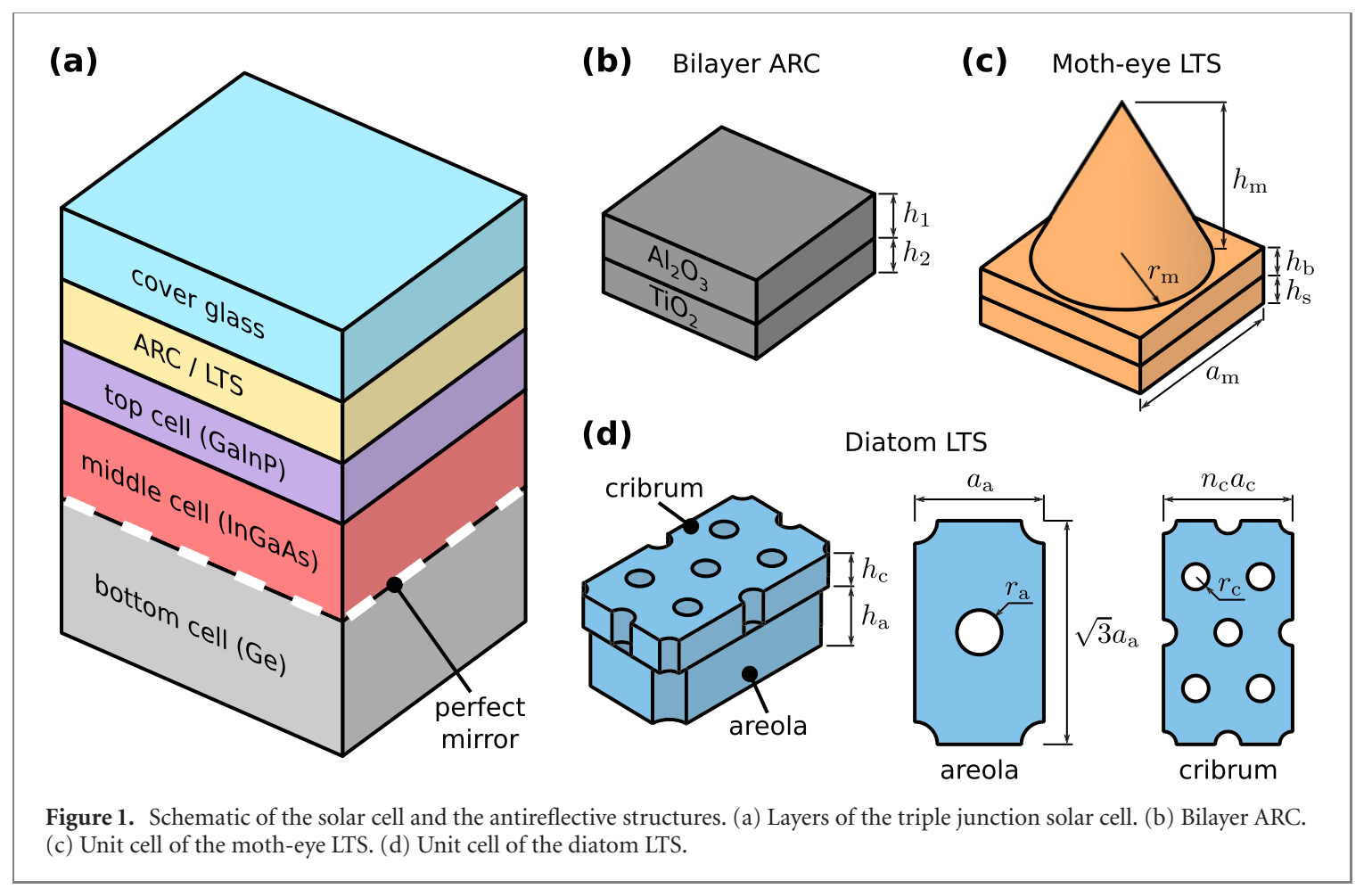

Table 1. Composition of layers used in the optical simulations, layer name and references to their optical properties.

\begin{tabular}{lcc}
\hline Material & Layer & Reference \\
\hline $\mathrm{Al}_{2} \mathrm{O}_{3}$ & Bilayer ARC & {$[43]$} \\
$\mathrm{TiO}_{2}$ & Bilayer ARC & {$[44]^{\mathrm{a}}$} \\
$\mathrm{Si}_{3} \mathrm{~N}_{4}$ & Diatom/moth-eye LTS & {$[45]$} \\
$\mathrm{Al}_{0.5} \mathrm{In}_{0.5} \mathrm{P}$ & Moth-eye LTS & {$[46]$} \\
$\mathrm{Ga}_{0.51} \mathrm{In}_{0.49} \mathrm{P}$ & Window & {$[47,48]$} \\
$\mathrm{In}_{0.01} \mathrm{Ga}_{0.99} \mathrm{As}$ & Top junction & {$[45]^{\mathrm{b}}$} \\
$\mathrm{Ge}$ & Middle junction & {$[49]$} \\
\hline
\end{tabular}

${ }^{a}$ Properties for polycrystalline anatase.

${ }^{\mathrm{b}}$ Optical properties of GaAs were used.

in nature and the spectrum of interest differs, therefore we have to redesign the structures to fit the requirements of the solar cell. The geometries of the LTS were parameterized and optimized, with the goal to achieve maximum solar cell efficiency. We compare the LTS performance to a reference ARC, which consists of two layers made of $\mathrm{Al}_{2} \mathrm{O}_{3}$ and $\mathrm{TiO}_{2}$ with thicknesses $h_{1}$ and $h_{2}$ as shown in figure 1(b).

The moth-eye structure is abstracted as a square lattice of cones, similar to $[39,40]$, with lattice constant $a_{\mathrm{m}}$. The unit cell is shown in figure 1(c). Each cone is described by the radius $r_{\mathrm{m}}$ and height $h_{\mathrm{m}}$ and it is placed on top of the base layer of thickness $h_{\mathrm{b}}$, which accommodates the guided modes that participate in the light trapping. The cones and the base layer are made of $\mathrm{TiO}_{2}$. A spacer layer of thickness $h_{\mathrm{s}}$, made out of $\mathrm{Si}_{3} \mathrm{~N}_{4}$ and placed between the base and the solar cell, adds an additional degree of freedom for the optimization.
The diatom LTS is based on the hard cell wall (frustule) of the diatom species Coscinodiscus wailesii described in [38]. The frustule of the C. wailesii is composed of three layers decorated by a hexagonal lattice of pores: the cribelum (top), cribrum (middle) and areola (bottom). The cribellum pore size is much smaller (around $50 \mathrm{~nm}$ ) than the typical wavelength of light; simulations of the individual layers have shown that the cribelum plays a minor role in the light enhancement and can be omitted [38]. In the present study, the diatom structure consists of two layers made of $\mathrm{TiO}_{2}$, resembling the areola and the cribrum, as shown in figure $1(\mathrm{~d})$. The unit cell of the areola layer is a rectangle with sides $a_{\mathrm{a}}$ and $\sqrt{3} a_{\mathrm{a}}$, perforated with holes of radii $r_{\mathrm{a}}$. The cribrum consists of an identical unit cell, which is scaled down by an integer factor in comparison to areola $a_{\mathrm{c}}=a_{\mathrm{a}} / n_{\mathrm{c}}$, $n_{\mathrm{c}} \geqslant 2, n_{\mathrm{c}} \in \mathbb{N}$. We denote the radius of the holes in the cribrum layer by $r_{\mathrm{c}}$. The two layers are displaced relative to each other by the displacement vector 
$r_{0} \in \mathbb{R}^{2}$; for $r_{0}=(0,0)$ the centers of all the areola holes coincide with hole centers of the cribrum. We denote the thickness of the areola and the cribum layers by $h_{\mathrm{a}}$ and $h_{\mathrm{c}}$ respectively.

\subsection{BOL solar cell model}

The propagation of light through the LTS structure and the solar cell was simulated using the Fourier modal method, also known as the scattering matrix method, a technique applicable to 3D layered structures with $2 \mathrm{D}$ periodicity. The absorption of wavelength $\lambda$ at junction $i \in\{\mathrm{GaInP}$ InGaAs, Ge $\}, a_{i}(\lambda)$, is computed by solving the scattering matrix for each layer, which connects the incoming light to the Poynting flux entering and exiting the layer bounds [42]. We sampled $a_{i}$ at 500 wavelengths between $350 \mathrm{~nm}$ and $1900 \mathrm{~nm}$. For all optical simulations, we assume that the cover glass layer has a constant refractive index of $n_{\text {cover }}=1.4$. For the remaining materials we consider a complex dispersive refractive index (see table 1 ). We include in the optical simulations the window layer of the top junction, an AlInP layer $15 \mathrm{~nm}$ thick that prevents the recombination of carriers at the top interface; the window layer is in direct contact with the LTS and therefore critical to the optical performance of the LTS due to the high refractive index contrast that introduces. Subsequent window and back surface field layers have a small refractive index contrast and have been lumped in the respective junctions. We model the SPM by increasing the dielectric constant of the Ge substrate to a very large value $\left(\sim 10^{9}\right)$ for photon energies $>1.4 \mathrm{eV}$, i.e. the gap of the middle junction. This creates a perfect dielectric mirror due to the high refractive index contrast. The number of basis functions for SSM was set to 51 per unit cell; for the diatom it translates to $51 n_{\mathrm{c}}$.

Given $a_{i}(\lambda)$, we estimate the BOL efficiency of the solar cell, $\eta_{0}$, by considering an equivalent electrical circuit. Each junction is abstracted as a current source, which accounts for the junction photocurrent, with a radiative coupling component and an ideal diode connected in parallel. At BOL, we make the simplifying assumption of complete photocarrier collection, which is reasonable for high quality III-V solar cells. Moreover, this definition of BOL efficiency serves as an informative figure of merit for the optical performance of the cell. Thus, the photocurrent density at junction $i$ becomes

$$
J_{\mathrm{ph}, i}=\frac{q}{h c} \int_{0}^{\lambda_{\mathrm{g}, i}} a_{i}(\lambda) \Phi_{\lambda}(\lambda) \lambda \mathrm{d} \lambda,
$$

where $q$ is the charge of the electron, $h$ is the Planck constant, $c$ is the speed of light and $\lambda_{\mathrm{g}, i}$ is the wavelength that corresponds to the bandgap of the junction. The spectral irradiance of the incident light, $\Phi_{\lambda}(\lambda)$, is computed using the AM0 spectrum. We include a radiative coupling model, which increases the photocurrent of the junction, if there is excess current in the junction above. The increase in current of the ith junction is computed using the recursion [50]

$$
J_{i}=\left\{\begin{array}{ll}
J_{\mathrm{ph}, i}+\beta \operatorname{ERE}\left(J_{i-1}-J\right) & J_{i-1}>J \\
J_{\mathrm{ph}, i} & \text { otherwise }
\end{array},\right.
$$

where $J$ is the common current flowing through the cell, ERE is the external radiative efficiency and $\beta$ is the radiative coupling parameter. No radiative coupling takes place in the first junction, namely $J_{\mathrm{GaInP}}=$ $J_{\mathrm{ph}, \mathrm{GaInP}}$, as the emission from the bottom cells is not absorbed by the higher bangap material of the top junction. Following [51], we assume constant values for $\beta=11$ and $\mathrm{ERE}=0.01$ and we compute the reverse saturation current density as

$$
\begin{aligned}
J_{0, i} & =\gamma_{i} J_{\mathrm{db}, i} / \text { ERE } \\
J_{\mathrm{db}, i} & =\frac{2 \pi q(k T)^{3}}{h^{3} c^{2}}\left[\left(\frac{E_{\mathrm{g}, i}}{k T}+1\right)^{2}+1\right] \mathrm{e}^{-E_{\mathrm{g}, i} / k T},
\end{aligned}
$$

where $k$ is the Boltzmann constant, $T$ the absolute temperature of interest and $E_{\mathrm{g}, i}$ is the band gap at the $i$ th junction. The coefficient $\gamma_{i}=1+\beta$ for junctions with the ideal SPM (top and middle in the present study) and $\gamma_{i}=1$ for the rest. The cell voltage is the sum of the voltage of each junction

$$
V=-R J+\sum_{i} \frac{k T}{q} \ln \left(\frac{J_{i}-J}{J_{0, i}}+1\right)
$$

with the series resistor $R=4 \times 10^{-5} \Omega \mathrm{m}^{2}$ lumping the contribution of all internal losses. We determine the maximum power per unit area numerically, $P_{\max }=\max (V J)$ and compute the BOL efficiency as:

$$
\eta_{0}=\frac{P_{\max }}{\int \Phi_{\lambda} \mathrm{d} \lambda} .
$$

\subsection{EOL solar cell model}

To connect the radiation exposure to the cell efficiency, we expanded the solar cell model to account for defect-induced changes in the semiconductor properties. Specifically, we assume that the exposure to radiation decreases the minority carrier lifetime from the initial value of $\tau_{0, a}$ to the value $\tau_{a}$ and increases the recombination velocity at the junction interface from $S_{0, a}$ to $S_{a}$. The subscript $a$ can refer either to electrons, $\mathrm{n}$, or holes, $\mathrm{p}$, depending on the type of doping. The relation of $\tau_{a}$ and $S_{a}$ with the radiation exposure is described in detail in the subsequent section 2.4. For the analysis, we neglect the radiation damage on the top and bottom junctions because the deterioration in solar cell performance is dominated by the degradation of the middle junction [52-54]. For the top and middle junctions we assume complete photocarrier collection.

The effect of the reduced lifetime of the solar cell efficiency is accounted for by expanding the BOL model to include $1 \mathrm{D}$ diffusion dynamics. Appendix A describes the 1D diffusion model and proposes an 
analytical solution. Two main adjustments are introduced in comparison to the complete photocarrier collection assumption. First, the photocurrent is limited by the collection probability, $P_{\mathrm{c}}$, which modifies (1) as

$$
\begin{aligned}
J_{\mathrm{ph}, \mathrm{InGaAs}}= & \frac{q}{h c} \int_{0}^{h_{\mathrm{InGaAs}}} \int_{0}^{\lambda_{\mathrm{g}}} P_{\mathrm{c}}(x) \alpha(\lambda, x) \\
& \times \Phi_{\lambda}(\lambda) \lambda \mathrm{d} \lambda \mathrm{d} x,
\end{aligned}
$$

where $x$ is the depth in the junction measured from the top, $h_{\text {InGaAs }}$ is the thickness of the middle junction and $\alpha(\lambda, x)$ denotes the derivative of the Poynting flux with respect to $x$, normalized by the flux entering the cell. Second, the junction saturation current increases due to the diffusion dynamics by the term $J_{\mathrm{d} 0}$ as

$$
J_{0, \text { InGaAs }}=\gamma J_{\mathrm{db}, \text { InGaAs }} / \mathrm{ERE}+J_{\mathrm{d} 0} .
$$

The calculation of the radiative coupling is performed as before, with the difference that $J_{\mathrm{ph}, \mathrm{InGaAs}}$ is calculated using (7). The EOL efficiency, $\eta$, is computed analogously to (6).

For the undamaged cell, we assume that $\tau_{0, a}$ is dominated by the radiation recombination lifetime and depends on the dopant concentrations as $\tau_{0, \mathrm{n}}=$ $\left(B N_{\mathrm{a}}\right)^{-1}$ and $\tau_{0, \mathrm{p}}=\left(B N_{\mathrm{d}}\right)^{-1}$, with $N_{\mathrm{a}}$ and $N_{\mathrm{d}}$ being the acceptor and donors concentrations respectively. The previous relations have been derived under the assumption that the majority carrier concentrations are approximately equal to the respective dopant concentrations.

\subsection{Radiation damage modeling}

The impact of radiation on $\tau_{a}$ and $S_{a}$ was assessed using Monte Carlo simulations. As high energy particles traverse through a material, they lose energy that falls into one of two categories [55, 56]: (a) electronic energy loss, which includes interactions of the projectile with the bound electrons of the medium and (b) nuclear energy loss, which accounts for the collisions between the projectile and the atoms of the medium. Semiconductor degradation is connected to a portion of the nuclear energy loss referred to as the nonionizing energy loss (NIEL). The deposited NIEL disturbs the nuclei off the semiconductor lattice, leading to the appearance of atomic displacements. Specifically, the energy deposited on atomic displacement per unit mass of the semiconductor, termed the damage dose $D_{\mathrm{d}}$, has been directly connected to the Frenkel pairs (FP, vacancy-interstitial pairs) density $[55,57]$.

The damage dose induced by a radiation source with spectral fluence $\phi$ (number of particles per area per energy) is equal to

$$
D_{\mathrm{d}}=\int \phi\left(E_{\mathrm{p}}\right) \frac{\mathrm{d} E_{\mathrm{de}}\left(E_{\mathrm{p}}\right)}{\mathrm{d} \chi} \mathrm{d} E_{\mathrm{p}},
$$

where $E_{\mathrm{p}}$ is the energy of the incoming particle and $\mathrm{d} E_{\mathrm{de}} / \mathrm{d} \chi$ is the displacement mass stopping power, namely the energy deposited on atomic displacements per unit path length normalized by the material density. Semiconductor atoms recoil with energy $E_{\mathrm{r}}$ after interaction with the particles and permanent defects occur if $E_{\mathrm{r}}$ is greater than a material dependent displacement threshold energy $E_{\mathrm{d}}$. Even if $E_{\mathrm{r}} \geqslant E_{\mathrm{d}}$, only part of $E_{\mathrm{r}}$ contributes to stable defect formation, captured by the Lindhard partition $L\left(E_{\mathrm{r}}\right)$, whereas the rest is dissipated as lattice vibrations $[58,59]$. Hence, the mass stopping power for all interaction is given as a function of $E_{\mathrm{p}}$ as

$$
\frac{\mathrm{d} E_{\mathrm{de}}\left(E_{\mathrm{p}}\right)}{\mathrm{d} \chi}=\frac{N_{\mathrm{A}}}{A} \sum_{k} \int_{E_{\mathrm{d}}}^{E_{\mathrm{M}}} E_{\mathrm{r}} L\left(E_{\mathrm{r}}\right) \frac{\mathrm{d} \sigma_{k}\left(E_{\mathrm{p}}, E_{\mathrm{r}}\right)}{\mathrm{d} E_{\mathrm{r}}} \mathrm{d} E_{\mathrm{r}},
$$

where $N_{\mathrm{A}}$ is the Avogadro number, $A$ is the atomic weight of the medium, $\mathrm{d} \sigma_{k} / \mathrm{d} E_{\mathrm{r}}$ is the differential cross section of the $k$ th reaction and $E_{\mathrm{M}}$ is the maximum recoil energy achievable by $E_{\mathrm{p}}$. The summation is taken over all possible reactions between the projectile and the semiconductor atoms. For protons and electrons interactions with the lattice atoms occur through Coulomb scattering. Additionally, for protons with energies above $\sim 10 \mathrm{MeV}$, nuclear elastic and inelastic reactions has to be taken into account [60]. Equation (10) is valid for simple substances, whereas for compound materials Bragg's rule applies; the mass stopping power is calculated as the sum of the mass stopping power of each material weighted by its mass fraction [52].

We employed the Geant4 software [61] to assess the impact of thinning the middle junction on $D_{\mathrm{d}}$ (see appendix B for simulation details). During the simulations, we track the particle path and record $E_{\mathrm{r}}$ of primary knock-on atoms (PKA, atoms that were displaced due to interaction with the energetic particles) in the middle junction. We set $E_{\mathrm{d}}=21 \mathrm{eV}$, value that has been proposed for GaAs $[52,54]$, and use the formulation in [58] for $L\left(E_{\mathrm{r}}\right)$. We approximate (9) as

$$
D_{\mathrm{d}}=\frac{\phi_{0}}{\phi m_{\mathrm{s}}} \sum_{n=1}^{N} E_{\mathrm{r}, n} L\left(E_{\mathrm{r}, n}\right),
$$

where $n$ is the event index, $N$ is the total number of recorded PKA events, $m_{\mathrm{s}}$ is the mass of the middle junction and $\phi_{0}$ is a reference fluence. The Frenkel pair concentration is computed by means of the modified Kinchin-Pease formula [55]

$$
\mathrm{FP} \sim \frac{D_{\mathrm{d}} \rho_{\mathrm{s}}}{E_{\mathrm{d}}},
$$

where $\rho_{\mathrm{s}}$ is the density of the middle junction material. We treat quantities that are computed from the Monte Carlo simulations as random variables and report the mean values with the $99 \%$ confidence interval $(\mathrm{CI})$. For $D_{\mathrm{d}}$, the mean value is computed from (11) and the variance from

$$
\sigma_{\mathrm{D}}^{2}=\left(\frac{\phi_{0}}{\phi m_{\mathrm{s}}}\right)^{2} \sum_{m=1}^{M}\left[E_{\mathrm{r}, m} L\left(E_{\mathrm{r}, m}\right)\right]^{2} .
$$


The distribution of $D_{\mathrm{d}}$ is assumed normal due to the large number of recorded events, similar to [62].

The FP density is connected to $\tau_{a}$ as follows: the increase in the inverse of the lifetime has been shown to be proportional to FP [55], and as a consequence of (12) is also proportional to $D_{\mathrm{d}}$. Therefore, after irradiation

$$
\frac{1}{\tau_{a}}=\frac{1}{\tau_{0, a}}+K_{a} D_{\mathrm{d}}
$$

where $K_{a}$ is the damage coefficient. We note that (14) is equivalent to the commonly used formula that connects the increase in the inverse of the lifetime to $\phi$ $[53,63]$. For the surface recombination velocity, we use the linear relation proposed in [63]

$$
S_{a}=S_{0, a}+K_{\mathrm{S} a} D_{\mathrm{d}}
$$

where $K_{\mathrm{S} a}$ is the interface damage rate.

\subsection{Geometry optimization}

For optimizing the LTS geometries, we are interested in attaining the maximum efficiency, $\eta$, while keeping the thickness of the middle junction, $h_{\mathrm{InGaAs}}$, to a minimum. These requirements form a multi-objective optimization problem:

$$
\begin{aligned}
& \min _{z_{\mathrm{p}}}\left(-\eta_{0}, h_{\mathrm{InGaAs}}\right) \\
& \text { s.t. } z_{\mathrm{p}} \in Z,
\end{aligned}
$$

where $z_{\mathrm{p}}$ is a vector holding the parameters of the LTS geometry. The set of allowable solutions, $Z$, incorporates the constraints on the parameters that encode meaningful solutions. We note that $Z$ can be extended to include additional constraints, like maximum or minimum allowable dimensions dictated by a given fabrication method. The solution to (16) is intended to findthe set of points, referred to as the Pareto front, that cannot be further improved without sacrificing at least one of the objectives.

We approximate the Pareto front using the software package vert pygmovert [64]. We employed the widely used evolutionary algorithm NSGA-II, which imitates the process of natural selection. Every distinct LTS geometry is seen as an 'individual', which is entirely described by its 'chromosome' $z_{\mathrm{p}}$. An initial population in $Z$ is selected randomly. At each step (generation), new individuals are created by crossover (mating) and mutation operations. Each new individual is assigned a fitness score, in our case the tuple $\left(-\eta_{0}, h_{\mathrm{InGaAs}}\right)$, and the next population is selected by taking the fitness score into account (survival of the fittest) as well as favoring solutions in underrepresented areas of the Pareto front (crowding distance). Different populations are allowed to evolve simultaneously and to exchange individuals during their evolution (island model with migration). The results for the diatom LTS were obtained using a single population of 52 individuals evolved for at least 270 generations. The moth-eye geometry instead was optimized by evolving four fully connected islands of 24 individuals each for 50 generations.

\section{Results}

\subsection{Optimized LTS}

Figure 2(a) compares the Pareto fronts for the diatom with $n_{\mathrm{c}}=2$ and moth-eye LTS to the bilayer ARC, as resulted from the optimization procedure. Both LTSs achieve higher $\eta_{0}$ values for thin middle junctions in comparison to the bilayer ARC. As $h_{\text {InGaAs }}$ increases, the efficiency gap between the moth-eye structure and the bilayer ARC diminishes, whereas $\eta_{0}$ for the diatom structure reaches a plateau at around $1 \mu \mathrm{m}$ of middle junction thickness. For the degradation analysis, it is informative to compare the different cell designs at fixed values of $h_{\text {InGaAs }}$. As a worst case scenario, we select for the moth-eye cell $h_{\text {InGaAs }}=1.13 \mu \mathrm{m}$, a thickness that achieves comparable BOL efficiency to the reference design. We set $h_{\text {InGaAs }}$ for the diatom LTS equipped cell at the same value as the moth-eye design to access any role of the optics in radiation degradation. The selected designs are marked in figure 2(a) by the points and the associated absorption profiles are contrasted in figure 2(b) for the wavelengths of interest of the middle junction.

We present the geometrical parameters of the optimized diatom and moth-eye structures in appendix C. For the bilayer ARC, the optimal layer thickness was determined to be relatively constant at $h_{1}=81 \mathrm{~nm}$ and $h_{2}=46 \mathrm{~nm}$. We further turn our attention to the diatom LTS, because the set of parameters introduced in section 2.1 does not encode all the possibilities and do not shed light on the importance of the cribrum layer. Thus, we repeated the optimizations for two additional configurations: a reversed ordering diatom, with the areola placed on top of the cribrum and a single dielectric layer with pores in a hexagonal lattice. We describe the latter configuration using the geometrical parameters of the areola, e.g. we use $a_{\mathrm{a}}$ for the lattice constant. Additionally, we performed the optimization with the normal ordering for the diatom including $n_{\mathrm{c}} \in[2,5]$ as an optimization parameter. The achieved maximum efficiency for the aforementioned configurations is shown in figure 3. The normal ordering is the most successful for wide range of $h_{\text {InGaAs. }}$. Only for very thin junctions, below $0.4 \mu \mathrm{m}$, is the reversed configuration able to achieve the highest efficiencies. Optimizations with $n_{\mathrm{c}}$ as a free parameter did not achieve substantially improved results in comparison to fixing $n_{\mathrm{c}}=2$. The single layer with pores attained the lowest $\eta_{0}$ for the vast range of $h_{\text {InGaAs }}$ or comparable for thick junctions.

\subsection{Radiation-induced damage}

The LTSs open the possibility of a substantial decrease in the thickness of the middle junction. To quantify the potential improvement in radiation hardness, we first assess the impact of junction thinning on 

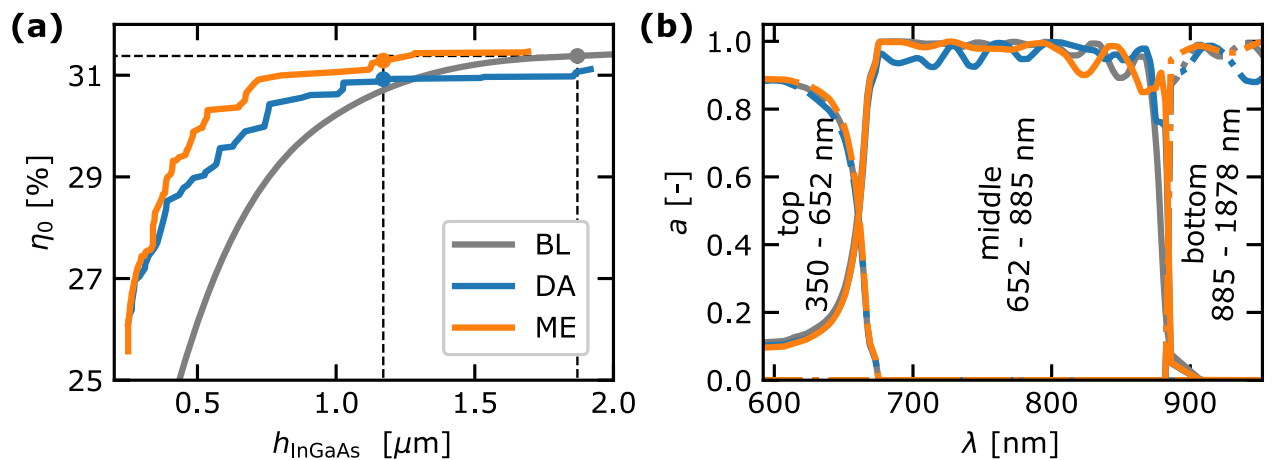

Figure 2. Optimization results for the LTS. (a) Comparison of the BOL efficiency between the LTS and the BL ARC. (b) Detail of the absorption of the middle junction per wavelength for the solar cells marked with points in panel (a). The wavelength ranges indicate the approximate absorption window of each subcell.

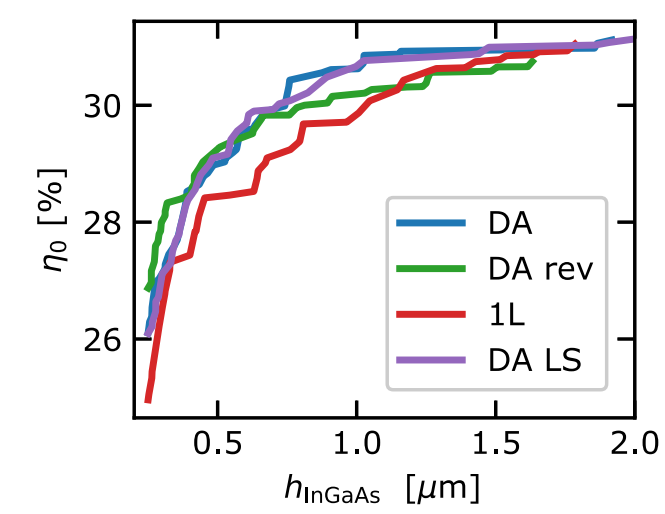

Figure 3. Optimal solar cell efficiency for different configurations of the diatom LTS. Four configurations are shown: normal layer ordering (DA), reversed ordering with the areola on top of the cribrum layer (DA rev), single layers with hexagonal pores $(1 \mathrm{~L})$ and normal layer ordering with $n_{\mathrm{c}}$ as an optimization parameter (DA LS).

$D_{\mathrm{d}}$ and consequently on the defect density. The relation between $D_{\mathrm{d}}$ and $h_{\text {InGaAs }}$ allows us to determine the decrease in the minority carrier lifetime for different cell designs at the same radiation exposure. To isolate the effects coming from the junction thinning alone, we simulated the damage dose deposition on middle junctions with thicknesses ranging from $0.2 \mu \mathrm{m}$ to $2.0 \mu \mathrm{m}$ for the bilayer ARC design. The material and thickness of the remaining cell layers were the same as the one used in the optical simulations, without the protective cover glass. The thickness of the bottom junction was fixed at $148 \mu \mathrm{m}$. Figures $4(\mathrm{a})$ and (b) plots $D_{\mathrm{d}}$ inflicted on the middle junction for monoenergetic electrons of $1 \mathrm{MeV}$ and $5 \mathrm{MeV}$, and protons of $3 \mathrm{MeV}$ and $10 \mathrm{MeV}$ at normal incidence; $D_{\mathrm{d}}$ depends on the energy and the type of the incident particle but not on the thickness of the middle junction. The independence of $D_{\mathrm{d}}$ from the junction thickness implies that for a given particle and energy, $\mathrm{d} E_{\mathrm{de}} / \mathrm{d} \chi$ remains almost constant as the particle traverses the middle junction. This is confirmed by the recorded values of $\mathrm{d} E_{\mathrm{de}} / \mathrm{d} \chi$ for the aforementioned cases, which are shown in figures 4 (c) and (d) for the two extreme cases of middle junction thickness.

Still, the dissimilar materials and thicknesses of the layers between the ARC and the LTS may affect the induced defect density. We repeat the simulation for the three cell designs, with the optimized LTS and reference ARC, which we discuss in section 3.1. We selected a representative space radiation environment, composed of electrons and protons meeting the solar cell at angles ranging from $0^{\circ}$ to $90^{\circ}$. Three different orbits were considered: a low-earth orbit (LEO), a highly elliptical orbit (HEO) and a geostationary orbit (GEO). Appendix B details the energy spectra the cells encounter in these orbits. All cells were simulated with a protective cover glass of $100 \mu \mathrm{m}$. Table 2 compares the $D_{\mathrm{d}}$ for the three solar cell designs, verifying that the solar cell degradation is connected to the radiation environment and not to the junction thickness or the LTS.

\subsection{EOL efficiency analysis}

The radiation-induced defect density on the middle junction is independent from the junction thickness and the ARC or the LTS, as discussed in section 3.2. Therefore, the deterioration in cell efficiency for different combinations of junction thickness and LTS can be compared at a given radiation exposure by computing $\eta$ at equal values of $\tau_{a}$ and $S_{a}$.

We assume a reference middle junction with geometry and doping similar to [63, device SB-SJ], with electrical characteristics that are summarized in table 3 (see also schematic in figure A1). We remark that the structure of the single junction devices in [63] include window layer and back surface field layer materials typically used in multijunction devices (AlInP and GaInP, respectively). Therefore the obtained bulk, surface and interface recombination parameters are applicable to the present case. Figure 5(a) compares the cell degradation at different radiation exposures for the three solar cell designs from section 3.1. We included the experimental $\eta$ values for the 3G30C cell [4] for comparison. The $\eta$ values for $3 \mathrm{G} 30 \mathrm{C}$ cell are reported for 
(a)
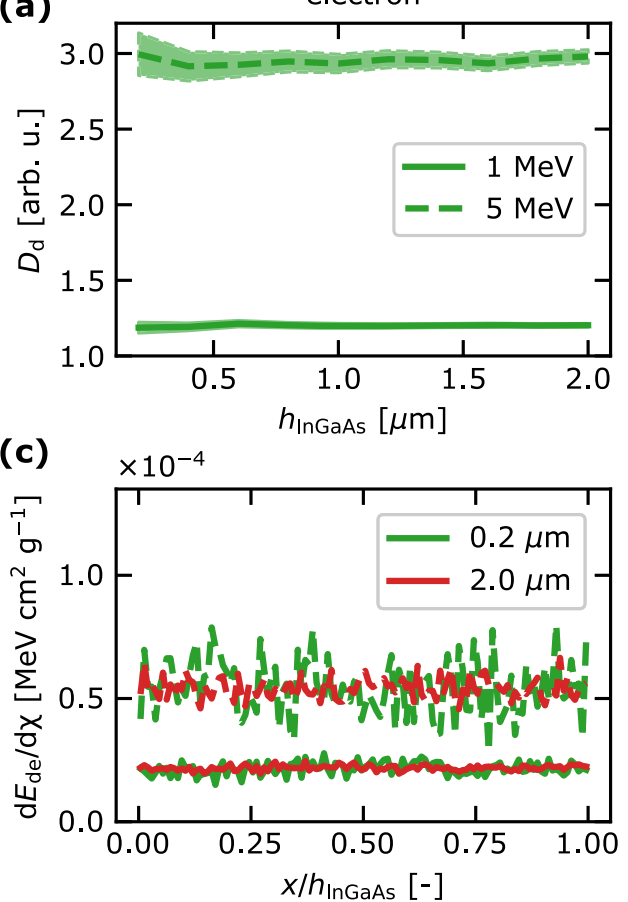

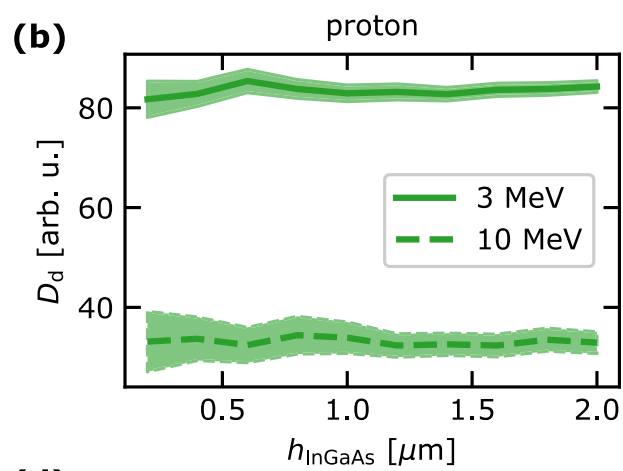

(d)

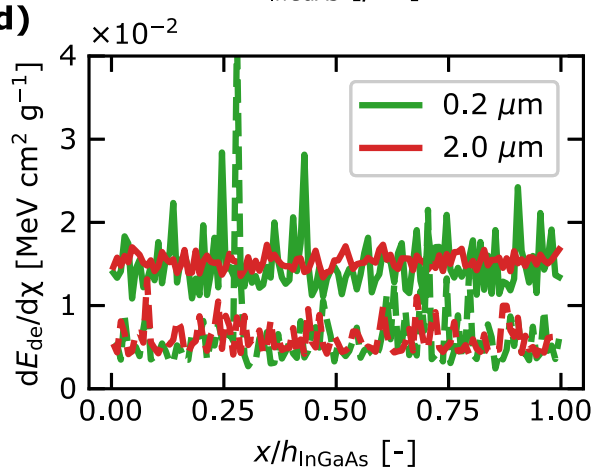

Figure 4. Radiation damage for different thicknesses of the middle junction. (a) Damage dose in arbitrary units deposited on the middle junction of a solar cell for incident electrons of $1 \mathrm{MeV}$ and $5 \mathrm{MeV}$. The middle junction thickness varies from $0.2 \mu \mathrm{m}$ to $2.0 \mu \mathrm{m}$, while all the other layers are kept constant. (b) Same as panel (a) for proton of $3 \mathrm{MeV}$ and $10 \mathrm{MeV}$. (c) Mass stopping power as measured along the normalized junction depth. The values correspond to the two extreme thickness of panel (a). Solid lines for $1 \mathrm{MeV}$, dashed lines for $5 \mathrm{MeV}$ electrons. CI has been omitted for the sake of clarity. (d) Similar to panel (c) for proton irradiation.

Table 2. Comparison of $D_{\mathrm{d}}\left(10^{9} \mathrm{MeV} \mathrm{g}^{-1}\right)$ between solar cells with the LTS (DA: diatom, ME: moth-eye) and the bilayer ARC (BL). Results for one year in orbit (LEO: low-earth, HEO: highly elliptical, GEO: geostationary) with $\phi_{0}$ equal to the total particle fluence.

\begin{tabular}{lcrr}
\hline Orbit & \multicolumn{1}{c}{ BL } & \multicolumn{1}{c}{ DA } & \multicolumn{1}{c}{ ME } \\
\hline LEO & $6.8 \pm 1.6$ & $7.0 \pm 2.0$ & $6.2 \pm 1.2$ \\
HEO & $1357.4 \pm 104.8$ & $1284.0 \pm 75.2$ & $1263.8 \pm 77.9$ \\
GEO & $40.2 \pm 30.4$ & $53.3 \pm 58.0$ & $35.7 \pm 16.1$ \\
\hline
\end{tabular}

$1 \mathrm{MeV}$ electron irradiation and the same applies for $K_{a}$ and $K_{\mathrm{S}, a}$ values: the conversion from particle fluence to $D_{\mathrm{d}}$ is performed with the coefficient $2.246 \times$ $10^{-5} \mathrm{MeV} \mathrm{cm}^{2} \mathrm{~g}^{-1}$ computed in section 3.2. Moreover, in figure 5(b) we plot the mean collection probability over the total width of the junction, $h_{\text {InGaAs }}$,

$$
P_{\mathrm{t}}=\frac{1}{h_{\mathrm{InGaAs}}} \int_{0}^{h_{\mathrm{InGaAs}}} P_{\mathrm{c}}(x) \mathrm{d} x
$$

which captures adequately the trends in $\eta$. The alteration of the electrical response of the solar cell due to the radiation exposure is exemplified in figure 6, which compares the current density versus the applied voltage between the pristine and the irradiated cells. The reduction in both the short-circuit current and the open-circuit voltage is evident for all designs.

The LTS equipped cells demonstrate higher radiation hardness, with $\eta$ being distinctly higher for exposure $D_{\mathrm{d}} \geqslant 10^{10} \mathrm{MeV} \mathrm{g}^{-1}$ in comparison to the
ARC design. The moth-eye cell retains a $1.8 \%$ to $2.6 \%$ higher efficiency than the reference design at high radiation doses, with the gain in $\eta$ being from $1.3 \%$ to $2.1 \%$ for the diatom LTS equipped cell at the same $D_{\mathrm{d}}$ range. Both LTS designs exhibit an identical degradation pattern, hinting that the absorption profile inside the junction influences the total photocurrent, but has little effect on the degradation dynamics. The simulated $\eta$ degradation shows a similar trend to the experimental results for the $3 \mathrm{G} 30 \mathrm{C}$ cell, without any fitting of the parameters. We further verified that the efficiency as a function of $D_{\mathrm{d}}$ follows the well known behavior described by the remaining factor, $\mathrm{RF}_{\eta}=\eta \eta_{0}^{-1}$,

$$
\mathrm{RF}_{\eta}=1-C \log _{10}\left(1+\frac{D_{\mathrm{d}}}{D_{\mathrm{x}}}\right)
$$

which has been used extensively in the literature $[5,52,54,65]$, with $C$ and $D_{\mathrm{x}}$ being fitting parameters. In all three cases we have found the coefficient of 
Table 3. Electrical properties of the middle junction used in the simulations.

\begin{tabular}{lcccc}
\hline Quantity & Symbol & Value & Units & Reference \\
\hline Donor concentration (emitter) & $N_{\mathrm{d}}$ & $3 \times 10^{18}$ & $\mathrm{~cm}^{-3}$ & {$[63]$} \\
Acceptor concentration (base) & $N_{\mathrm{a}}$ & $3 \times 10^{16}$ & $\mathrm{~cm}^{-3}$ & {$[63]$} \\
Emitter width & $\bar{w}_{\mathrm{n}}$ & 0.1 & $\mu \mathrm{m}$ & {$[63]$} \\
Recombination velocity (p-doped) & $S_{0, \mathrm{p}}$ & 100 & $\mathrm{~cm} \mathrm{~s}^{-1}$ & {$[63]$} \\
Recombination velocity (n-doped) & $S_{0, \mathrm{n}}$ & 360 & $\mathrm{~cm} \mathrm{~s}^{-1}$ & {$[63]$} \\
Radiative recombination rate & $B$ & $2 \times 10^{-10}$ & $\mathrm{~cm}^{3} \mathrm{~s}^{-1}$ & {$[53]$} \\
Intrinsic carrier concentration & $n_{\mathrm{i}}$ & $2 \times 10^{6}$ & $\mathrm{~cm}^{-3}$ & {$[53]$} \\
Dielectric constant & $\epsilon_{\mathrm{s}}$ & $1.16 \times 10^{-10}$ & $\mathrm{~F} \mathrm{~m}^{-1}$ & \\
Damage coefficient (p-doped) & $K_{\mathrm{p}}$ & 0.04 & $\mathrm{~g} \mathrm{MeV}^{-1} \mathrm{~s}^{-1}$ & {$[63]$} \\
Damage coefficient (n-doped) & $K_{\mathrm{n}}$ & 0.23 & $\mathrm{~g} \mathrm{MeV}^{-1} \mathrm{~s}^{-1}$ & {$[63]$} \\
Interface damage rate (p-doped) & $K_{\mathrm{Sp}}$ & $7.6 \times 10^{-7}$ & $\mathrm{~g} \mathrm{~cm} \mathrm{MeV}^{-1} \mathrm{~s}^{-1}$ & {$[63]$} \\
Interface damage rate (n-doped) & $K_{\mathrm{Sn}}$ & 0 & $\mathrm{~g} \mathrm{~cm} \mathrm{MeV}^{-1} \mathrm{~s}^{-1}$ & {$[63]$} \\
\hline
\end{tabular}
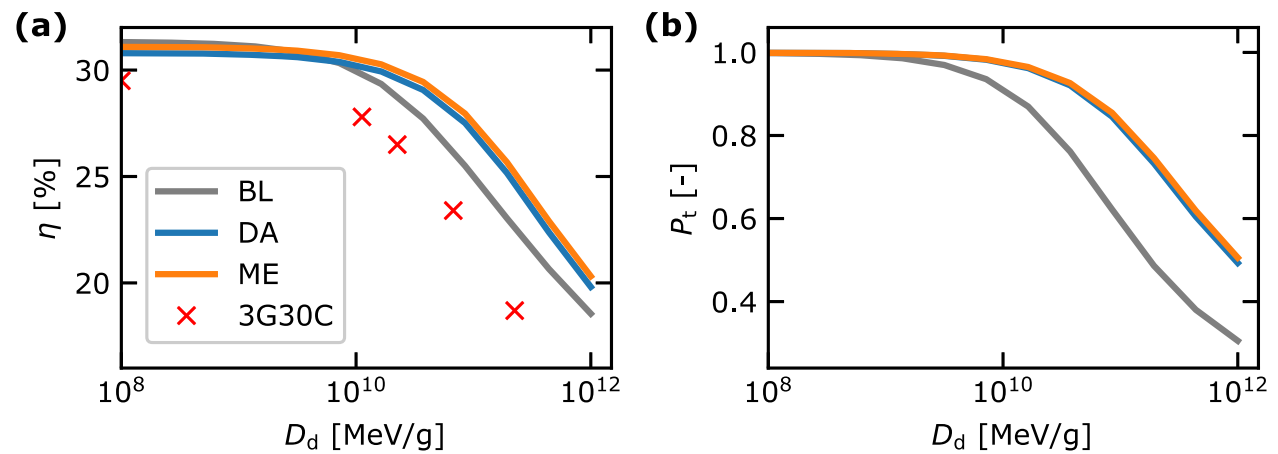

Figure 5. Comparison of EOL efficiency at different $D_{\mathrm{d}}$. (a) Efficiency deterioration for various levels of $D_{\mathrm{d}}$ for different cells designs (BL for bilayer ARC, DA for diatom LTS, ME for moth-eye LTS). Values for the 3G30C cell from [4] are included for comparison. (b) Mean collection probability over the junction width for the same cases as panel (a).

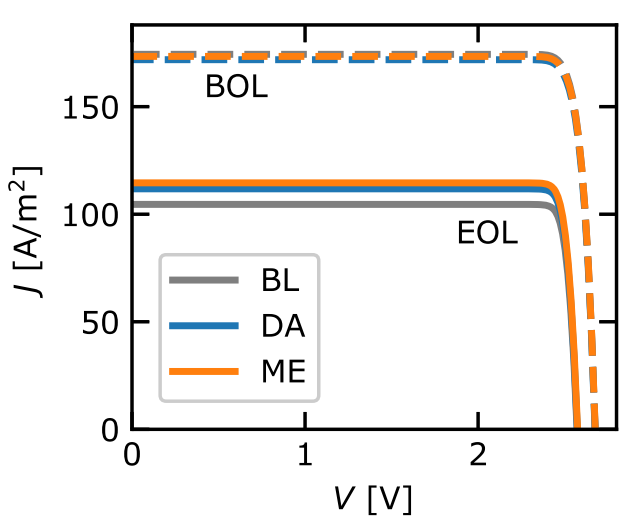

Figure 6. $J-V$ curves at BOL and EOL conditions. Current density versus cell voltage for the three solar cells designs (BL for bilayer ARC, DA for diatom LTS, ME for moth-eye LTS) at BOL (dashed lines, no radiation exposure) and EOL ( solid lines, $D_{\mathrm{d}}=10^{12} \mathrm{MeV} \mathrm{g}^{-1}$ ).

determination $R^{2}>0.999$, illustrating the validity of our model.

So far we have looked into the LTS and junction thickness pairs that achieve comparable BOL to the reference design. For applications where the EOL alone is of interest, enhanced radiation harness can be attained at the cost of BOL efficiency. To quantify the BOL efficiency to radiation hardness tradeoff, we simulated the EOF efficiency of the solar cell designs depicted in figure 2 at a medium $\left(D_{\mathrm{d}}=2 \times\right.$ $\left.10^{10} \mathrm{MeV} \mathrm{g}^{-1}\right)$ and high $\left(D_{\mathrm{d}}=10^{11} \mathrm{MeV} \mathrm{g}^{-1}\right)$ radiation exposure. For all cells junctions we used the same electrical characteristics as before, given in table 3. Figure 7 plots the efficiency of the optimized bilayer ARC and LTS for a wide range of $h_{\text {InGaAs }}$. At any given $D_{\mathrm{d}}$, there is an optimal middle junction thickness for the bilayer ARC and the LTS cells, which balances the gains of increased photocarrier generation due to absorption to the losses due to the diffusion-driven collection. At this particular thickness, the efficiency after irradiation is the highest, even when compared to cells with significantly higher BOL efficiency. At the tested $D_{\mathrm{d}}$, the diatom and the moth-eye LTS were able to achieve from $0.4 \%$ to $1.2 \%$ and from $0.8 \%$ to $1.9 \%$ higher efficiency than the bilayer ARC respectively. The bilayer ARC design displayed the highest sensitivity with respect to the middle junction thickness where the maximum $\eta$ appears, moving from $1.28 \mu \mathrm{m}$ at $D_{\mathrm{d}}=2 \times 10^{10} \mathrm{MeV} \mathrm{g}^{-1}$ to $0.87 \mu \mathrm{m}$ at $D_{\mathrm{d}}=10^{11} \mathrm{MeV} \mathrm{g}^{-1}$. For the same $D_{\mathrm{d}}$ values, the diatom LTS design is less sensitive with the maxima appearing at $1.06 \mu \mathrm{m}$ and $0.76 \mu \mathrm{m}$, whereas the motheye LTS with $h_{\text {InGaAs }}=0.72 \mu \mathrm{m}$ achieved consistently the highest $\eta$. Therefore, both LTSs enable the solar cell to operate closer to the optimum $\eta$ for a wider range of radiation exposures than the bilayer ARC by properly selecting $h_{\text {InGaAs }}$. 

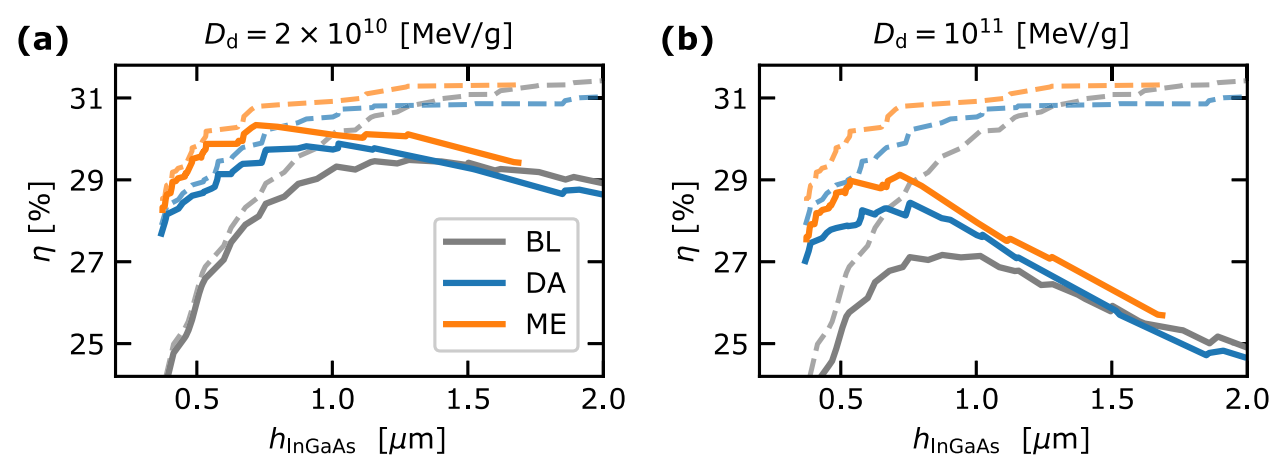

Figure 7. Comparison of efficiency degradation for various cell designs at medium and high radiation exposure. (a) Efficiency versus middle junction thickness for cells of figure 2 at $D_{\mathrm{d}}=2 \times 10^{10} \mathrm{MeV} \mathrm{g}^{-1}$ (BL for bilayer ARC, DA for diatom LTS, ME for moth-eye LTS). Dashed lines indicate the BOL efficiency. (b) Similar to panel (a) for $D_{\mathrm{d}}=10^{11} \mathrm{MeV} \mathrm{g}^{-1}$.

\section{Discussion}

The LTS, inspired by the moth-eye and the diatom patterns, increases the efficiency of the solar cell in comparison to the bilayer ARC commonly used in industry for cells with a thin middle junction. The bilayer ARC improves light absorption by decreasing the reflectance of the front surface, minimizing the optical losses. The LTS further boosts the efficiency by diffracting light which, in combination with the SPM, couples at the guided modes of the middle junction. The diatom LTS incorporates two photonic crystals of different lattice constant, opening more diffraction orders due to the combined effect of the stack. The higher enhancement in $\eta_{0}$ observed for the normal and the reversed diatom configurations, in comparison to the single perforated layer, can be attributed to this fact. These results reflect that the two-fold diffraction introduced by two stacked photonic crystals is indeed very beneficial to enhance the efficiency of the solar cell above the single layer case. Chen et al [38] showed for an ultra-thin $(50 \mathrm{~nm})$ organic absorber that the total enhancement is the combination of each individual photonic crystal contribution, which agrees with our results. In the reverse order, the large lattice constant $a_{\mathrm{a}}$ is facing the front medium which might introduce reflection losses. This can explain the reduction in efficiency for the thick layers (see figure 3), where diffraction is not as important as the anti-reflection mechanism. For thin InGaAs, the efficiency of both orderings are similar, being even higher for the thinnest cases studied. This again is in line with the results of [38], as the authors show a similar enhancement for the reversed order for the ultra-thin absorber. Still, the enhancement due to the diatom LTS is comparable to the moth-eye structure only for $h_{\text {InGaAs }}$ smaller than approximately $0.4 \mu \mathrm{m}$.

Aside from the solar cell performance, the adoption of light trapping technologies is also governed by cost considerations. The bilayer ARC, composed of two simple layers, is definitely the simplest to manufacture. Progress in manufacturing processes has made large-scale production of 3D structures possible. The moth-eye cone pattern has been transferred to $\mathrm{Si}_{3} \mathrm{~N}_{4}$ by laser interference lithography [40], a low cost technique capable of uniformly patterning large areas [66]. Moreover, nanocone arrays have been manufactured out of $\mathrm{Si}[67], \mathrm{TiO}_{2}[68,69]$ and glass [37] using plasma and dry etching. The double photonic crystal arrangement of the diatom structure makes its assembly a challenging task. Precise porous hexagonal arrays, resembling the single diatom layer, have been fabricated using the photochemical etching technique [70], but bonding of the two layers is necessary to complete the diatom structure. A fabrication process is explained in [71] for a square lattice, although the authors recognize the challenge it represents. The implementation of the SPM poses similar issues. A selective reflective mirror can be realized as a DBR. For the DBR to achieve the necessary wavelength bandwidth (the absorption window of the middle junction), the optical design requires the use of either thick structures with hundreds of layers or materials with a high refractive index contrast. Additionally, the extinction coefficient of the DBR material should be as low as possible to avoid any parasitic losses. These requirements can be met with either thick graded buffer Bragg reflectors [14], or by using, for example, a dielectric $\mathrm{SiO}_{2} / \mathrm{TiO}_{2} \mathrm{DBR}$ [72] The former increases the final price in its actual form, and the latter is incompatible with III-V epitaxy reactors. Therefore, there is a separate and necessary task in identifying a DBR structure that could combine lower price and monolithic growth. Combining the structures presented in this work with an optimized DBR will impact positively on radiation-hard solar cells for space applications, and lower the material usage and cost of III-V multijunctions, fundamental also for future terrestrial applications.

According to our simulations, the radiation damage sustained by thin cell junctions in space is mainly governed by the radiation environment. Taking into account the energy deposition mechanism, this is a reasonable observation. The energy of the incident particle dictates the rate of energy loss along the 
trajectory through a given material. For highly energetic particles the total energy deposit is small compared to the initial energy, since the solar cell structure is relatively thin. Therefore, the energy of the particle remains almost constant along its path and so does the deposited energy per unit path length, resulting in an almost constant $D_{\mathrm{d}}$. There are cases where localized energy deposit may take place in the semiconductor material, like for low energy protons that can be stopped inside the junction with a Bragg peak produced at the end of the track. The continuous energy spectrum of the typical space environment and the wide range of incident angles would diminish any localized effect. This conclusion of approximately uniform damage distribution throughout the cell active region is in agreement with [73], where omnidirectional proton irradiation was analyzed.

Thinning of the middle junction, when combined with the absorption enhancement of the LTS, provides a compelling way to increase the robustness and the radiation hardness of multi-junction solar cells. Sacrificing BOL efficiency allows for optimal operation of the cell for a wider range of $D_{\mathrm{d}}$. Although not examined in the present study, reduction in junction thickness should be accompanied by systematic tuning of the junction electrical characteristics, such as emitter width and doping concentration, to extort the maximum possible improvement with respect to cell radiation hardness. Previous studies have addressed these issues; the emitter width has been connected to the radiation hardness of solar cells, with shallow emitters exhibiting slower degradation [63]. Lowering the base doping concentrations has a positive effect on radiation hardness, as suggested by $[9,53]$.

From the mission design point of view, the thickness of the protective cover glass has been the single design parameter to control the radiation degradation for space solar cells, an approach that has been extensively discussed previously $[5,74]$. The cover glass thickness affects the sustained damage by altering the energy spectrum perceived by the solar cell material through shielding. Reducing the sensitivity of the cells to radiation is a complementary approach to shielding and both methods can be applied simultaneously. This can result in reduction of the array weight, either from reducing the protective glass thickness or the number of panels needed to achieve the EOL power requirements.

\section{Conclusion}

In the present study, we reported an indirect biomimetic approach to increase the radiation hardness of space solar cells; the model organisms are not directly related to the radiation problem but provide solutions to the issues arising from adopting designs that are insensitive to radiation cells. To this end, we employed comprehensive models to analyze the behavior of multi-junction ( $\mathrm{GaInP} / \mathrm{InGaAs} / \mathrm{Ge}$ ) solar cells starting from their optical performance and their interaction with radiation-induced defects. Then, we incorporated the optical- and radiation-related results in an electrical model which predicts the cell efficiency and allows us to directly compare different junction and LTS designs. We showed that thinning of the middle junction, when accompanied by optical compensation, provides an attractive approach to developing radiation hardened and robust solar cells. Specifically, our proposal enables an increase of up to $2 \%$ in EOL efficiency in comparison to the currently used cell design, which in turn can lead to a significant reduction in the weight of spaceborne solar panel arrays.

Future work includes addressing fabrication issues in more detail and assessing potential costs associated with the large scale manufacture of such structures.

\section{Acknowledgments}

The authors thank Katharina Hildebrandt (ACTESA) for kicking the project off and for her spadework. The authors acknowledge the support of CESGA for the provision of the computing resources, specially for the computing time on FinisTerrae II. Funding was provided by the Spanish Government and the European Union through AEIFEDER-UE (ENE2017-91092-EXP, RTI2018-096937B-C22, RYC-2017-21995) and Comunidad de Madrid (P2018/EMT-4308).

\section{Data availability statement}

The data that support the findings of this study are available upon reasonable request from the authors.

\section{Appendix A. 1D diffusion model}

The modeling approach of the present section is based on the analytical model of $\mathrm{p}-\mathrm{n}$ homojunctions and the underlying assumptions found in $[75$, section 4.4]. The main difference is that in the present analysis we consider an arbitrary absorption profile inside the junction, which is described by $\alpha(\lambda, x)$, in contrast to the commonly assumed exponential profile based on the Beer-Lambert law. This is crucial in order to capture the effect of the LTS on the junction photocurrent.

We discuss a junction composed of an n-type emitter with donor density $N_{\mathrm{d}}$ connected and a ptype base with acceptor density $N_{\mathrm{a}}$. The junction is divided into three regions, as shown in figure A1; the n-type quasi-neutral area (QNA, emitter), the p-type QNA (base) and the depletion region. The width of the emitter and base is $\bar{w}_{\mathrm{p}}$ and $\bar{w}_{\mathrm{n}}$ respectively. The depletion area has its center at $x_{\mathrm{W}}$ from the 


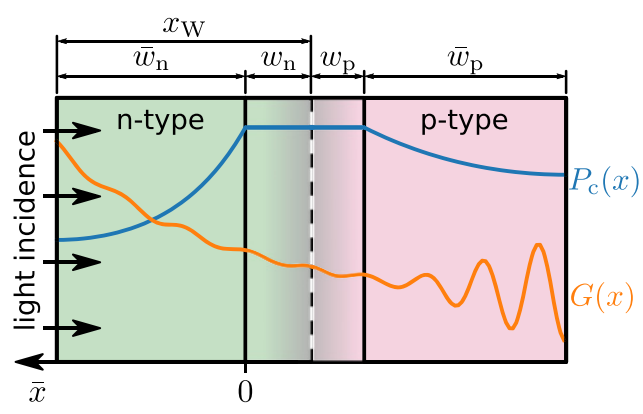

Figure A1. Schematic of the $\mathrm{p}-\mathrm{n}$ junction used in the 1D diffusion model.

light entering face and extends by $w_{\mathrm{p}}$ and $w_{\mathrm{n}}$ toward the two QNAs. In the following, we use the coordinate system $\bar{x}$, which has its origin at the interface between the depletion region and the n-type QNA (see figure A1)

At steady state, the constant current density, $J$, is the result of the sum of the electron, $J_{n}$, and hole, $J_{\mathrm{p}}$ current densities. Since $J$ is constant throughout the structure, it can be evaluated at any given plane. For convenience, we perform the computation at the plane $\bar{x}=0$, i.e. at the interface between the depletion region and the n-type QNA.

$$
J=J_{\mathrm{p}}(0)+J_{\mathrm{n}}(0)
$$

The hole current density at this plane is related to the excess minority carrier density, $\delta p$, as [75]

$$
J_{\mathrm{p}}(0)=-q \mathcal{D}_{\mathrm{p}} \frac{\mathrm{d} \delta p(0)}{\mathrm{d} \bar{x}},
$$

where $q$ is the elementary charge $(e)$ and $\mathcal{D}_{\mathrm{p}}$ is the hole diffusion constant.

First we turn our attention to the n-type QNA, where $\delta p$ follows the diffusion equation

$$
\frac{\mathrm{d}^{2} \delta p}{\mathrm{~d} \bar{x}^{2}}-\frac{1}{L_{\mathrm{p}}^{2}} \delta p+G(\bar{x})=0,
$$

where $L_{\mathrm{p}}=\sqrt{\mathcal{D}_{\mathrm{p}} \tau_{\mathrm{p}}}$ is the minority carrier diffusion length. The generation rate is given, similarly to (1), as

$$
G(\bar{x})=\frac{\mathcal{D}_{\mathrm{p}}}{h c} \int_{0}^{\lambda_{\mathrm{g}}} \alpha(\lambda, \bar{x}) \Phi_{\lambda}(\lambda) \lambda \mathrm{d} \lambda .
$$

Two boundary conditions accompany (A.3):

$$
\begin{array}{cc}
\frac{\mathrm{d} \delta p\left(\bar{w}_{\mathrm{n}}\right)}{\mathrm{d} \bar{x}}=-\frac{S_{\mathrm{p}}}{\mathcal{D}_{\mathrm{p}}} \delta p\left(\bar{w}_{\mathrm{n}}\right) & \bar{x}=\bar{w}_{\mathrm{n}} \\
\delta p(0)=p_{0}\left(\mathrm{e}^{\frac{E_{\mathrm{Fp}}}{k T}}-1\right) & \bar{x}=0,
\end{array}
$$

where $p_{0}$ is the equilibrium hole density, $E_{\mathrm{Fp}}$ is the quasi-Fermi level and $S_{\mathrm{p}}$ is the rear surface recombination velocity. We obtain the solution to (A.3) using the Green's function approach. The Green's function satisfies

$$
\frac{\mathrm{d}^{2} \mathcal{G}(\bar{x}, s)}{\mathrm{d} \bar{x}^{2}}-\frac{1}{L_{\mathrm{p}}^{2}} \mathcal{G}(\bar{x}, s)=\delta(\bar{x}-s)
$$

with $\delta(\bar{x}-s)$ Dirac's $\delta$ function. The general solution to (A.7) is of the form

$\mathcal{G}(\bar{x}, s)=\left\{\begin{array}{ll}c_{1}(s) \cosh \left(\frac{\bar{x}}{L_{\mathrm{p}}}\right)+c_{2}(s) \sinh \left(\frac{\bar{x}}{L_{\mathrm{p}}}\right) & \bar{x}<s \\ c_{3}(s) \cosh \left(\frac{\bar{x}}{L_{\mathrm{p}}}\right)+c_{4}(s) \sinh \left(\frac{\bar{x}}{L_{\mathrm{p}}}\right) & \bar{x} \geqslant s\end{array}\right.$,

where the coefficients $c_{1}$ to $c_{4}$ depend solely on $s$. At the point $\bar{x}=s, \mathcal{G}(\bar{x}, s)$ is continuous and satisfies (A.7), resulting in

$$
\begin{aligned}
& c_{1}-c_{3}=L_{\mathrm{p}} \sinh \left(\frac{s}{L_{\mathrm{p}}}\right) \\
& c_{4}-c_{2}=L_{\mathrm{p}} \cosh \left(\frac{s}{L_{\mathrm{p}}}\right) .
\end{aligned}
$$

Given $\mathcal{G}(\bar{x}, s)$, the excess minority carrier density becomes

$$
\begin{aligned}
\delta p(\bar{x}) & =-\int_{0}^{\bar{w}_{\mathrm{n}}} \mathcal{G}(\bar{x}, s) G(s) \mathrm{d} s \\
& =\bar{c}_{1} \cosh \left(\frac{\bar{x}}{L_{\mathrm{p}}}\right)+\bar{c}_{2} \sinh \left(\frac{\bar{x}}{L_{\mathrm{p}}}\right)-(\mathcal{K} * G)(\bar{x}),
\end{aligned}
$$

where $\bar{c}_{1}=-\int_{0}^{\bar{w}_{\mathrm{n}}} c_{1} G \mathrm{~d} s$ and $\bar{c}_{2}=-\int_{0}^{\bar{w}_{\mathrm{n}}} c_{2} G \mathrm{~d} s$. The last term in (A.11) stands for the convolution of the kernel $\mathcal{K}=L_{\mathrm{p}} \sinh \left(\bar{x} L_{\mathrm{p}}^{-1}\right)$ with the generation rate

$$
(\mathcal{K} * G)(\bar{x})=\int_{0}^{\bar{x}} L_{\mathrm{p}} \sinh \left(\frac{\bar{x}-s}{L_{\mathrm{p}}}\right) G(s) \mathrm{d} s .
$$

Likewise, the derivative of $\delta p$ with respect to $\bar{x}$ becomes

$\frac{\mathrm{d} \delta p}{\mathrm{~d} \bar{x}}=\frac{\bar{c}_{1}}{L_{\mathrm{p}}} \sinh \left(\frac{\bar{x}}{L_{\mathrm{p}}}\right)+\frac{\bar{c}_{2}}{L_{\mathrm{p}}} \cosh \left(\frac{\bar{x}}{L_{\mathrm{p}}}\right)-\left(\frac{\mathrm{dK}}{\mathrm{d} \bar{x}} * G\right)(\bar{x})$.

The remaining constants can be determined by applying the boundary conditions:

$$
\begin{aligned}
\bar{c}_{1}= & p_{0}\left(\mathrm{e}^{\frac{V}{k T}}-1\right) \\
\bar{c}_{2}= & \frac{L_{\mathrm{p}}}{c_{\mathrm{p}}}\left[(\mathcal{P} * G)\left(\bar{w}_{\mathrm{n}}\right)-\frac{\bar{c}_{1}}{L_{\mathrm{p}}} \sinh \left(\frac{\bar{w}_{\mathrm{n}}}{L_{\mathrm{p}}}\right)\right. \\
& \left.-\frac{\bar{c}_{1} S_{\mathrm{p}}}{\mathcal{D}_{\mathrm{p}}} \cosh \left(\frac{\bar{w}_{\mathrm{n}}}{L_{\mathrm{p}}}\right)\right] \\
c_{\mathrm{p}}= & \cosh \left(\frac{\bar{w}_{\mathrm{n}}}{L_{\mathrm{p}}}\right)+\frac{S_{\mathrm{p}} L_{\mathrm{p}}}{\mathcal{D}_{\mathrm{p}}} \sinh \left(\frac{\bar{w}_{\mathrm{n}}}{L_{\mathrm{p}}}\right) \\
\mathcal{P}(\bar{x})= & \frac{\mathrm{dK}(\overline{\mathrm{x}})}{\mathrm{d} \bar{x}}+\frac{S_{\mathrm{p}}}{\mathcal{D}_{\mathrm{p}}} \mathcal{K}(\bar{x}),
\end{aligned}
$$

where we have substituted $E_{\mathrm{Fp}}=V$ under the assumption that the quasi-Fermi level is constant 
throughout the depletion region [75]. The hole current density is computed from (A.2) and (A.13),

$$
J_{\mathrm{p}}=-\frac{q \mathcal{D}_{\mathrm{p}}}{L_{\mathrm{p}}} \bar{c}_{2} .
$$

Two contributions can be identified in $\bar{c}_{2}$. The first comes from the photocurrent, which is computed as the integral of the generation rate in the QNA multiplied by the collection probability

$$
P_{\mathrm{c}}(\bar{x})=c_{\mathrm{p}}^{-1} \mathcal{P}\left(\bar{w}_{\mathrm{n}}-\bar{x}\right) .
$$

The last two terms depend on the applied voltage and contribute to the diode saturation current.

A similar analysis can be performed in the p-region to compute $J_{\mathrm{n}}(0)$. However, it is more convenient to compute this current contribution at the interface between the p-type QNA and the depletion region, $\bar{x}=-w_{\mathrm{p}}-w_{\mathrm{n}}$. The current density at $\bar{x}=0$ is obtained from the continuity condition:

$$
J_{\mathrm{n}}(0)=J_{\mathrm{n}}\left(-w_{\mathrm{p}}-w_{\mathrm{n}}\right)+J_{\mathrm{R}},
$$

where $J_{R}$ is the current density accounting for the recombination losses in the depletion region. We assume that $J_{\mathrm{R}}$ is independent of $\tau_{\mathrm{p}}$ and $\tau_{\mathrm{n}}$ and it is given from (3). See [75] for further details.

Adding the current density of the QNAs to the one of depletion area, where we assume complete photocarrier collection, the total photocurrent is computed as in (7) with

$$
P_{\mathrm{c}}(x)= \begin{cases}c_{\mathrm{p}}^{-1}\left[\cosh \left(\frac{x}{L_{\mathrm{p}}}\right)+\frac{S_{\mathrm{p}} L_{\mathrm{p}}}{\mathcal{D}_{\mathrm{p}}} \sinh \left(\frac{x}{L_{\mathrm{p}}}\right)\right] & 0 \leqslant x \leqslant \bar{w}_{\mathrm{n}} \\ 1 & \bar{w}_{\mathrm{n}}<x<h_{i}-\bar{w}_{\mathrm{p}} \\ c_{\mathrm{n}}^{-1}\left[\cosh \left(\frac{h_{i}-x}{L_{\mathrm{n}}}\right)+\frac{S_{\mathrm{n}} L_{\mathrm{n}}}{\mathcal{D}_{\mathrm{n}}} \sinh \left(\frac{h_{i}-x}{L_{\mathrm{n}}}\right)\right] & h_{i}-\bar{w}_{\mathrm{p}} \leqslant x \leqslant h_{i},\end{cases}
$$

where $h_{i}$ is the total width of the junction, $\mathcal{D}_{\mathrm{n}}$ is the electron diffusion constant, $S_{\mathrm{n}}$ is the front surface recombination velocity and

$$
c_{\mathrm{n}}=\cosh \left(\frac{\bar{w}_{\mathrm{p}}}{L_{\mathrm{n}}}\right)+\frac{S_{\mathrm{n}} L_{\mathrm{n}}}{\mathcal{D}_{\mathrm{n}}} \sinh \left(\frac{\bar{w}_{\mathrm{p}}}{L_{\mathrm{n}}}\right) .
$$

The origin of the coordinate system in (A.21) has been moved to the light-entering face of the junction, to be in accordance with (7). The increase in the saturation current is obtained by the sum of the contribution of each QNA, $J_{\mathrm{d} 0}=J_{\mathrm{d} 0, \mathrm{n}}+J_{\mathrm{d} 0, \mathrm{p}}$, with

$$
\begin{aligned}
& J_{\mathrm{d} 0, \mathrm{n}}=\frac{q \mathcal{D}_{\mathrm{n}} n_{0}}{c_{\mathrm{n}} L_{\mathrm{n}}}\left[\sinh \left(\frac{\bar{w}_{\mathrm{p}}}{L_{\mathrm{n}}}\right)+\frac{S_{\mathrm{n}} L_{\mathrm{n}}}{\mathcal{D}_{\mathrm{n}}} \cosh \left(\frac{\bar{w}_{\mathrm{p}}}{L_{\mathrm{n}}}\right)\right] \\
& J_{\mathrm{d} 0, \mathrm{p}}=\frac{q \mathcal{D}_{\mathrm{p}} p_{0}}{c_{\mathrm{p}} L_{\mathrm{p}}}\left[\sinh \left(\frac{\bar{w}_{\mathrm{n}}}{L_{\mathrm{p}}}\right)+\frac{S_{\mathrm{p}} L_{\mathrm{p}}}{\mathcal{D}_{\mathrm{p}}} \cosh \left(\frac{\bar{w}_{\mathrm{n}}}{L_{\mathrm{p}}}\right)\right],
\end{aligned}
$$

where $n_{0}$ is the equilibrium minority carrier concentration. We point out that for negligible recombination velocity at the edges of the junction, $L_{\mathrm{p}} \gg \bar{w}_{\mathrm{p}}$ and $L_{\mathrm{n}} \gg \bar{w}_{\mathrm{n}}$, we retrieve the original model with $P_{\mathrm{c}}=1$ throughout the junction and no contribution to the saturation current from the QNAs.

The values for $\mathcal{D}_{\mathrm{p}}$ and $\mathcal{D}_{\mathrm{n}}$ for GaAs were computed using the relations from [76] under the assumption that the minority carrier mobility is equal the majority carrier mobility at any given region. The parameters of the depletion region were determined using the abrupt junction approximation under the assumption that the majority carrier concentration is equal to the dopant concentration. Specifically, the equilibrium densities were computed as $p_{0}=n_{\mathrm{i}}^{2} N_{\mathrm{d}}^{-1}$ and $n_{0}=n_{\mathrm{i}}^{2} N_{\mathrm{a}}^{-1}$, where $n_{\mathrm{i}}$ is the intrinsic carrier concentration. The depletion region width is calculated from

$$
w_{\mathrm{p}}=\sqrt{\frac{2 \epsilon_{\mathrm{s}} k T}{q^{2}} \frac{N_{\mathrm{d}}}{N_{\mathrm{a}}} \frac{1}{N_{\mathrm{d}}+N_{\mathrm{a}}} \ln \left(\frac{N_{\mathrm{d}} N_{\mathrm{a}}}{n_{\mathrm{i}}^{2}}\right)}
$$

and $w_{\mathrm{n}}=N_{\mathrm{d}}^{-1} N_{\mathrm{a}} w_{\mathrm{p}}$, where $\epsilon_{\mathrm{s}}$ is the dielectric constant. The previous relation neglects any shrinkage of the depletion region due to the applied voltage.

\section{Appendix B. Geant4 validation and simulation details}

All Geant4 simulations were performed using the dedicated physics list for the space radiation environment (QBBC) [77], with the multiple scattering models being substituted by a single scattering one at the middle junction. It is possible to track the PKAs in the semiconductor material, only if the dedicated single Coulomb scattering physics $[56,78]$ are selected in the physics list. The single scattering model though is computationally more expensive than the commonly used multiple scattering model [79] and its use is restricted when the highest possible accuracy is needed.

We are interested in the PKAs occurring in the middle junctions, which constitutes less than $1.5 \%$ 
Table B1. Comparison of $D_{\mathrm{d}}$ [arb.u.] for single (SSC) and combined single/multiple scattering (MSC) models.

\begin{tabular}{lcc}
\hline Source & SSC & MSC \\
\hline $1 \mathrm{MeV}$ electron & $0.021 \pm 0.002$ & $0.020 \pm 0.002$ \\
$5 \mathrm{MeV}$ electron & $0.054 \pm 0.009$ & $0.052 \pm 0.008$ \\
$3 \mathrm{MeV}$ proton & $14.875 \pm 0.671$ & $14.918 \pm 0.683$ \\
$10 \mathrm{MeV}$ proton & $6.084 \pm 1.200$ & $6.154 \pm 1.058$ \\
\hline
\end{tabular}
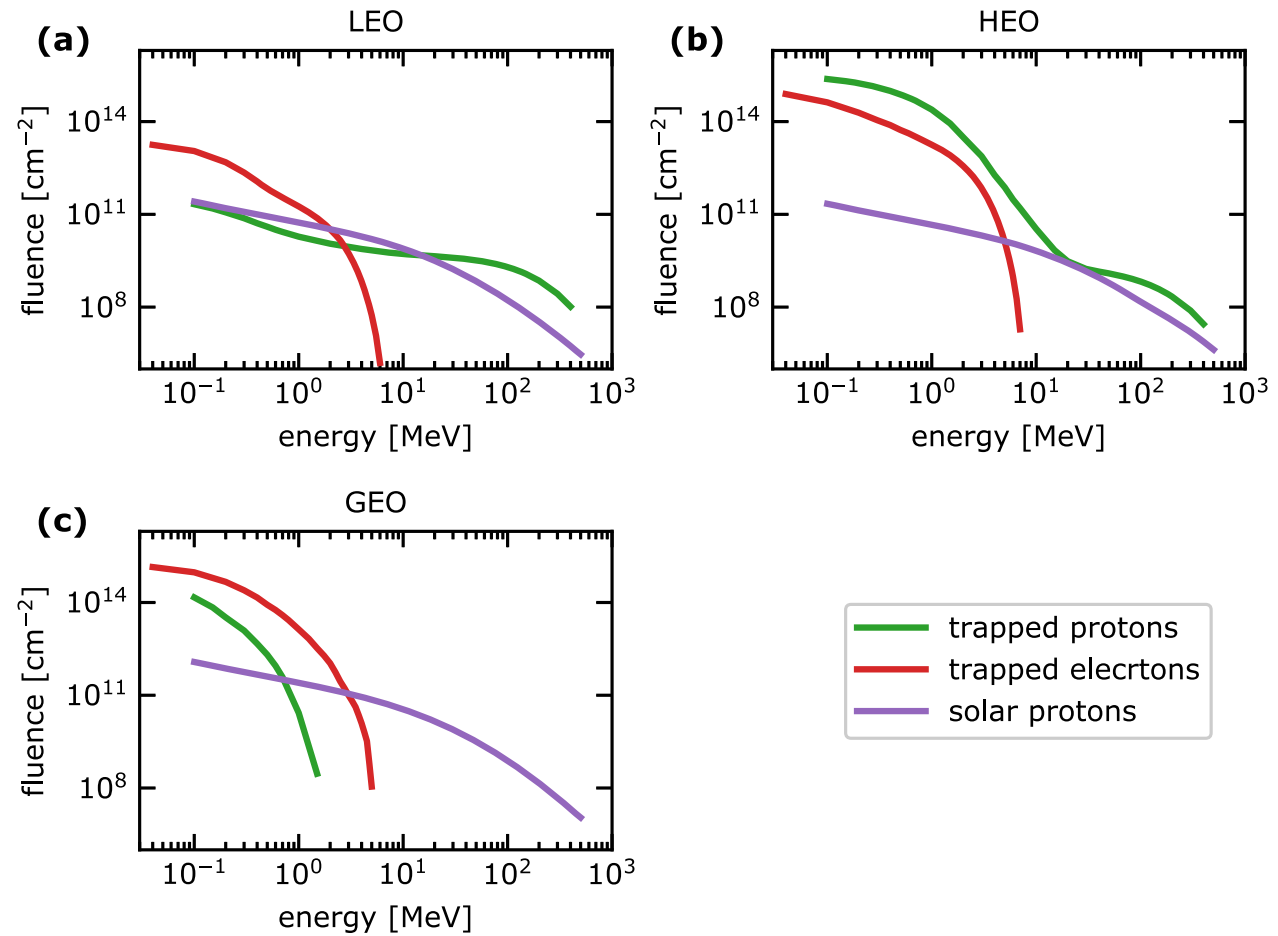

Figure B1. Integral particle fluence over the course of one year for three different orbits. (a) LEO at $800 \mathrm{~km}$ with $98.0^{\circ}$ inclination. (b) HEO at $700 \mathrm{~km} \times 12050 \mathrm{~km}$ with $63.4^{\circ}$ inclination. (c) GEO.

of the total thickness of the solar cell stack. To keep the simulations' time within reasonable limits, we combine the multiple and single scattering models in our code. The layers of the solar cell are grouped in two regions: (a) layers where the $D_{\mathrm{d}}$ needs to be calculated and (b) the remaining layers. For the first region, the single scattering model was selected and the secondary production range cut was set to $40 \mathrm{~nm}$, whereas for the second region the multiple scattering is used and the secondary production range cut was set to $400 \mathrm{~nm}$. We validated the code by comparing the simulations results to the ones with uniform single scattering model and $40 \mathrm{~nm}$ secondary production range cut over the whole cell geometry. Table B1 contrasts the $D_{\mathrm{d}}$ values from the two implementations for monoenergetic $1 \mathrm{MeV}$ and $5 \mathrm{MeV}$ electron, and $3 \mathrm{MeV}$ and $10 \mathrm{MeV}$ proton sources with incident normal to the solar cell for $10^{6}$ primary events. The difference between the computed values lie inside the CI bounds. The relative speedup for the single/multiple scattering combined physics list was measured between 41 and 77 times.
A realistic space radiation environment can be constructed for given Earth trajectories. The spectrum contains three contributions: trapped electron and protons, and solar protons. In our simulations we considered three orbits: a LEO at $800 \mathrm{~km}$ with $98.0^{\circ}$ inclination, a HEO at $700 \mathrm{~km} \times 12050 \mathrm{~km}$ with $63.4^{\circ}$ inclination and a GEO. The trapped particle spectrum was determined using the AP- 8 and AE- 8 models and the solar proton spectrum was calculated from ESP total fluence model, using the software SPENVIS [80]. The integral particle fluence over the time span of one year is shown in figure B1.

\section{Appendix C. Geometrical parameters for optimized LTS}

The optimal geometrical parameters of the three structures studied in figure 3 are gathered in figures $\mathrm{C} 1(\mathrm{a})-(\mathrm{d})$. For the single layer, the optimal $a_{\mathrm{a}}$ assumes roughly two values: around $0.60 \mu \mathrm{m}$ for $h_{\text {InGaAs }}<0.80 \mu \mathrm{m}$ and $0.35 \mu \mathrm{m}$ for $h_{\text {InGaAs }}>0.80 \mu \mathrm{m}$. This implies that the scattering of light is playing an important role in the former case. Wavelengths 

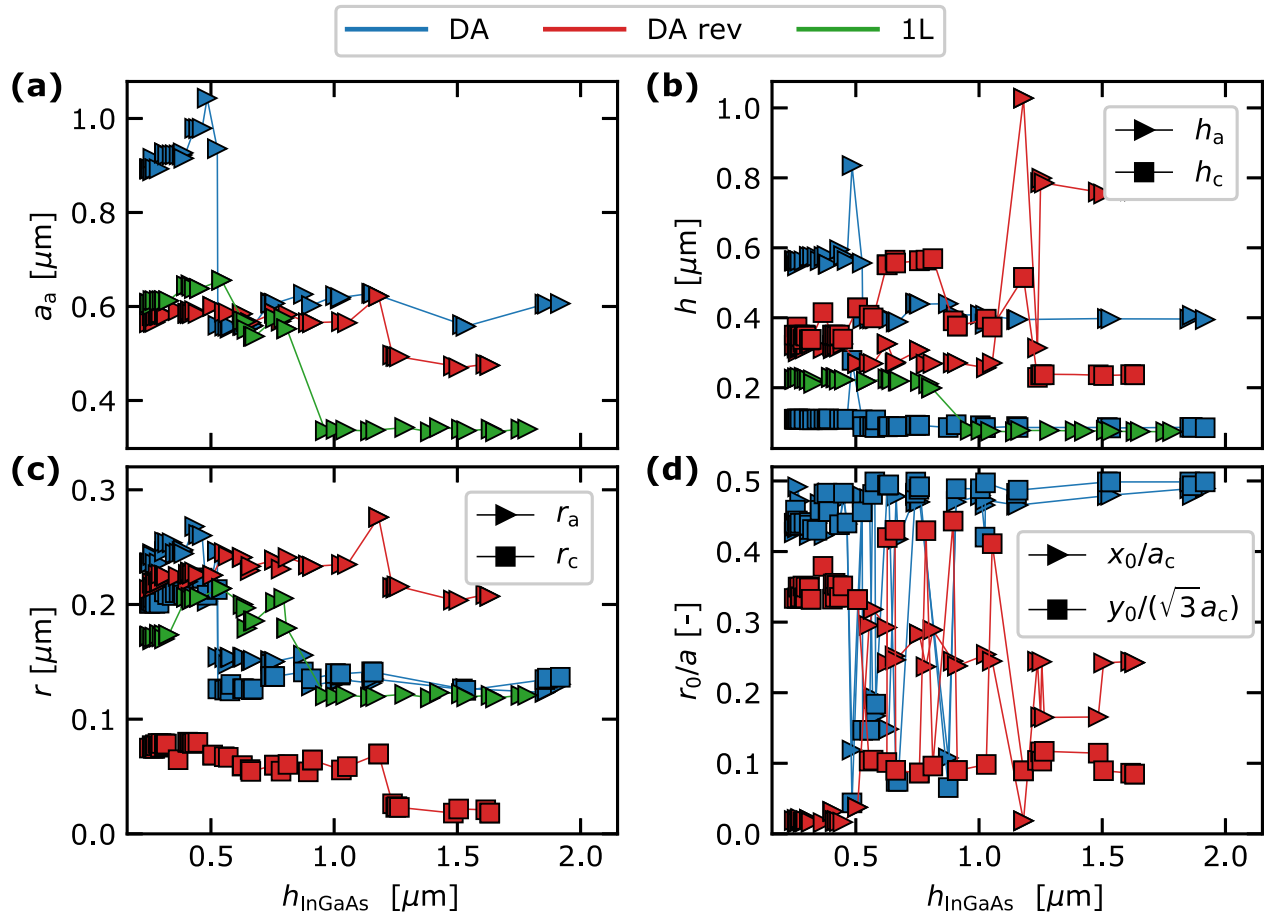

Figure C1. Geometrical parameters for the optimized diatom structures. Parameters for three configurations are shown: normal layer ordering (DA), reversed ordering (DA rev) and single layers with hexagonal pores (1L).
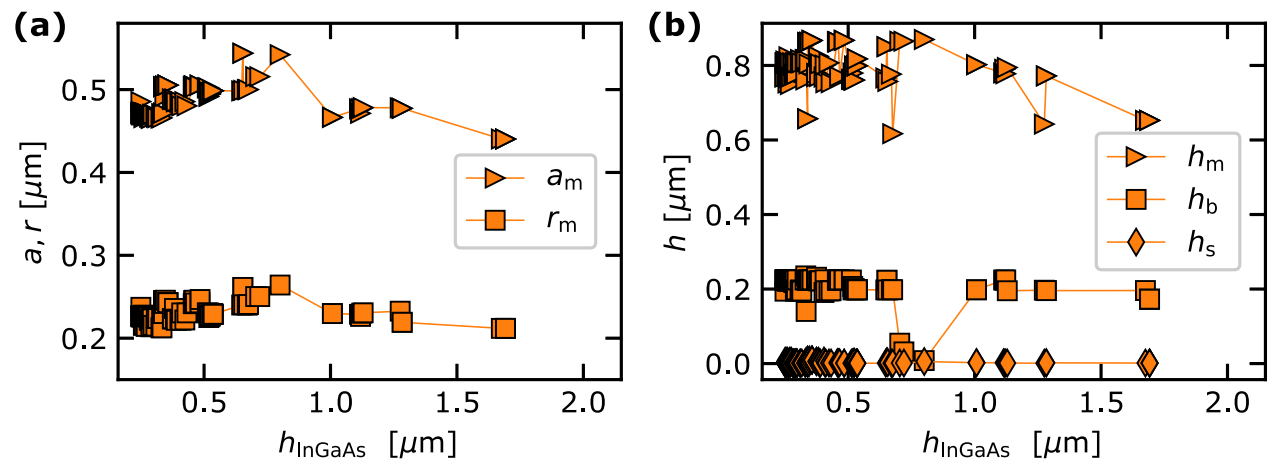

Figure C2. Geometrical parameters for the optimized moth-eye structures.

smaller than $a_{\mathrm{a}} / n_{\text {cover }}$ can be back-diffracted hence introducing reflection losses. In contrast, diffraction inside of the cell is beneficial for light-trapping. A trade-off is established between both processes. Light-trapping is critical for thin absorbers to expand the optical path length and, therefore, $a_{\mathrm{a}}$ is larger than in the case of thick ones. A similar split into two distinct regions is observed for the single layer $h_{\mathrm{a}}$ and $r_{\mathrm{a}}$ parameters. This means that the average refractive index is higher as holes reduce, i.e. that the single layer acts more as a single layer ARC than as a light-trapping element for thick InGaAs. This is also supported by the reduction of the $h_{\mathrm{a}}$ approaching toward the typical thickness of an interferential filter.

For the diatom configuration, $a_{\mathrm{a}}$ follows a similar pattern at almost double the value of the single layer, with the transition taking place at $h_{\mathrm{InGaAs}}=0.50 \mu \mathrm{m}$. That means that the cribum lattice constant takes a value very close to that of the single layer. Likewise, the thickness and the pore radius shows the same trend as $a_{\mathrm{a}}$. As the thickness of the middle junction increases, $r_{\mathrm{a}}$ and $r_{\mathrm{c}}$ converge to the value of $0.12 \mu \mathrm{m}$, which coincides with the pore radius for the single layer. The relative displacement between the two layers plays a secondary role, with the optimized values exhibiting a spread; there is a slight preference for a constant value toward the diagonal of the unit cell, with $x_{0} / a_{c}$ and $y_{0} /\left(\sqrt{3} a_{\mathrm{c}}\right)$ both adopting values close to 0.5 . For the reversed diatom configuration, two regions are present with the transition happening at $h_{\text {InGaAs }}=$ $1.20 \mu \mathrm{m}$. The relative displacement in this case seems to play a secondary role in the $\eta_{0}$ enhancement. The geometry of cribrum layer for diatom configuration 
and the single layer are similar for thick junctions, implying that the areola layer plays a supporting role on the first.

The parameters for the optimized moth-eye (ME) LTS are shown in figure C2. The lattice constant $a_{\mathrm{m}}$ shows a softer evolution than $a_{\mathrm{a}}$ with $h_{\text {InGaAs. The }}$ cones help in balancing the trade-off between reflection losses and diffracted light if compared with the hole arrangement constituting the diatom structure. Indeed, the ME can be seen as an averaged graded index layer, which helps in reducing the impedance mismatch between the solar cell layers and the cover glass. The radius is always larger than $0.2 \mu \mathrm{m}$ which means that the cone base tends to completely fill the unit cell area, i.e. $r_{\mathrm{m}} / a_{\mathrm{m}} \approx 0.5$. The cone height $h_{\mathrm{m}}$ takes very large values, close to $800 \mathrm{~nm}$ in the region of interest, i.e. for thin middle junctions. The base and spacer thicknesses $h_{\mathrm{b}}$ and $h_{\mathrm{s}}$ are almost constant along the whole optimization range, the latter being almost negligible. If $h_{\mathrm{c}}$ is added to $h_{\mathrm{m}}$ we get the total amount of $\mathrm{TiO}_{2}$ needed to fabricate the structure. A layer of $1 \mu \mathrm{m}$ is indeed needed. This is an experimental challenge, as high index and high quality $\mathrm{TiO}_{2}$ is difficult to obtain beyond $100 \mathrm{~nm}$. More research and alternative proposals will be addressed in the future to cope with this issue.

\section{ORCID iDs}

Thomas Vasileiou (D) https://orcid.org/0000-00029342-7948

José M Ripalda (D) https://orcid.org/0000-0003-32338308

Dario Izzo (D) https://orcid.org/0000-0002-98468423

\section{References}

[1] Dawson S, Stella P, McAlpine W and Smith B 2012 JUNO photovoltaic power at Jupiter 10th Int. Energy Conversion Engineering Conf. (IECEC) (American Institute of Aeronautics and Astronautics)

[2] ESA 2019 ESA's Technology Strategy for Space19+

[3] Bett A W et al 2009 Highest efficiency multi-junction solar cell for terrestrial and space applications 24th European Photovoltaic Solar Energy Conference (Munich: WIP) pp 1-6

[4] Guter W, Dunzer F, Ebel L, Hillerich K, Köstler W, Kubera T, Meusel M, Postels B and Wächter C 2017 Space solar cells-3G30 and next generation radiation hard products E3S Web Conf. 1603005

[5] Messenger S R, Jackson E M, Warner J H and Walters R J 2010 SCREAM: a new code for solar cell degradation prediction using the displacement damage dose approach 2010 35th IEEE Photovoltaic Specialists Conf. (IEEE) pp 001106-11

6] Hirst L C, Yakes M K, Warner J H, Bennett M F, Schmieder K J, Walters R J and Jenkins P P 2016 Intrinsic radiation tolerance of ultra-thin GaAs solar cells Appl. Phys. Lett. 109 033908

[7] Hirst L 2019 Ever thinner high-efficiency cells Nat. Energy 4 $726-7$
[8] Polly S J, Nelson G T, D’Rozario J R, Tatavarti R and Hubbard S M 2019 Radiation effects in thinned GaAs photovoltaics incorporating DBRs for improved radiation tolerance of multijunctions 2019 IEEE 46th Photovoltaic Specialists Conf. (PVSC) pp 2818-21

[9] Maximenko S I, Lumb M P, Moore J, Hirst L C, Yakes M K and Jenkins P P 2019 Thin GaAs solar cells for high irradiation levels 46th Photovoltaic Specialists Conf. (PVSC) pp 2814-7

[10] Cariou R et al 2019 Radiation hardness of ultra-thin GaAs solar cells with rear-side silver mirror 46th Photovoltaic Specialists Conf. (PVSC) pp 2811-3

[11] Yamaguchi M 2001 Radiation-resistant solar cells for space use Sol. Energy Mater. Sol. Cells 68 31-53

[12] Chen H-L et al 2019 A 19.9\%-efficient ultrathin solar cell based on a $205 \mathrm{~nm}$-thick GaAs absorber and a silver nanostructured back mirror Nat. Energy 4 761-7

[13] Buencuerpo J, Steiner M A and Tamboli A C 2020 Optically-thick $300 \mathrm{~nm}$ GaAs solar cells using adjacent photonic crystals Opt. Express 28 13845-60

[14] France R M, Espinet-González P, Ekins-Daukes N J, Guthrey H, Steiner M A and Geisz J F 2018 Multijunction solar cells with graded buffer Bragg reflectors IEEE $J$. Photovolt. 8 1608-15

[15] Bozzola A, Liscidini M and Andreani L C 2012 Photonic light-trapping versus Lambertian limits in thin film silicon solar cells with 1D and 2D periodic patterns Opt. Express 20 A224-44

[16] Bermel P, Luo C, Zeng L, Kimerling L C and Joannopoulos J D 2007 Improving thin-film crystalline silicon solar cell efficiencies with photonic crystals Opt. Express 1516986

[17] Yu Z, Raman A and Fan S 2010 Fundamental limit of nanophotonic light trapping in solar cells Proc. Natl Acad. Sci. USA 107 17491-6

[18] Brongersma M L, Cui Y and Fan S 2014 Light management for photovoltaics using high-index nanostructures Nat. Mater. 13 451-60

[19] Mokkapati S and Catchpole K R 2012 Nanophotonic light trapping in solar cells J. Appl. Phys. 112101101

[20] Saeta P N, Ferry V E, Pacifici D, Munday J N and Atwater H A 2009 How much can guided modes enhance absorption in thin solar cells? Opt. Express 17 20975-90

[21] van Lare M-C and Polman A 2015 Optimized scattering power spectral density of photovoltaic light-trapping patterns ACS Photonics 2 822-31

[22] Burresi M, Pratesi F, Riboli F and Wiersma D S 2015 Complex photonic structures for light harvesting $A d v$. Opt. Mater. 3 722-43

[23] Essig S et al 2017 Raising the one-Sun conversion efficiency of III-V/Si solar cells to $32.8 \%$ for two junctions and $35.9 \%$ for three junctions Nat. Energy 217144

[24] Simon J, Schulte K L, Horowitz K A W, Remo T, Young D L and Ptak A J 2019 III-V-based optoelectronics with low-cost dynamic hydride vapor phase epitaxy Crystals 93

[25] Lombardero I, Ochoa M, Miyashita N, Okada Y and Algora C 2020 Theoretical and experimental assessment of thinned germanium substrates for III-V multijunction solar cells Prog. Photovolt. Res. Appl. 28 1097-106

[26] Han Z, Xu J, Liu X, Zhang H, Wang D, Chen Z, Zhang D and Fan T 2018 Bio-inspired photonic materials: prototypes and structural effect designs for applications in solar energy manipulation Adv. Funct. Mater. 281705309

[27] Parker A R and Townley H E 2007 Biomimetics of photonic nanostructures Nat. Nanotechnol. 2 347-53

[28] Fuhrmann T, Landwehr S, El Rharbi-Kucki M and Sumper M 2004 Diatoms as living photonic crystals Appl. Phys. B 78 257-60

[29] De Stefano L, Rea I, Rendina I, De Stefano M and Moretti L 2007 Lensless light focusing with the centric marine diatom Coscinodiscus walesii Opt. Express 15 18082-8

[30] De Tommasi E, Rea I, Mocella V, Moretti L, De Stefano M, Rendina I and De Stefano L 2010 Multi-wavelength study of 
light transmitted through a single marine centric diatom Opt. Express 18 12203-12

[31] Romann J, Valmalette J-C, Chauton M S, Tranell G, Einarsrud M-A and Vadstein O 2015 Wavelength and orientation dependent capture of light by diatom frustule nanostructures Sci. Rep. 517403

[32] Stavenga D G, Foletti S, Palasantzas G and Arikawa K 2006 Light on the moth-eye corneal nipple array of butterflies Proc. R. Soc. B 273 661-7

[33] Ko D-H, Tumbleston J R, Henderson K J, Euliss L E, DeSimone J M, Lopez R and Samulski E T 2011 Biomimetic microlens array with antireflective 'moth-eye' surface Soft Matter 7 6404-7

[34] Han Z, Jiao Z, Niu S and Ren L 2019 Ascendant bioinspired antireflective materials: opportunities and challenges coexis Prog. Mater. Sci. 103 1-68

[35] Dewan R, Fischer S, Benno Meyer-Rochow V, Özdemir Y, Hamraz S and Knipp D 2011 Studying nanostructured nipple arrays of moth eye facets helps to design better thin film solar cells Bioinspiration Biomimetics 7016003

[36] Chiadini F, Fiumara V, Scaglione A and Lakhtakia A 2010 Simulation and analysis of prismatic bioinspired compound lenses for solar cells Bioinspiration Biomimetics 5026002

[37] Min Song Y, Jeong Y, Yeo C I and Lee Y T 2012 Enhanced power generation in concentrated photovoltaics using broadband antireflective coverglasses with moth eye structures Opt. Express 20 A916-23

[38] Chen X, Wang C, Baker E and Sun C 2015 Numerical and experimental investigation of light trapping effect of nanostructured diatom frustules Sci. Rep. 511977

[39] Buencuerpo J, Llorens J M, Dotor M L and Ripalda J M 2015 Broadband antireflective nano-cones for tandem solar cells Opt. Express 23 A322-36

[40] Buencuerpo J, Torné L, Álvaro R, Llorens J M, Dotor M L and Ripalda J M 2016 Nano-cones for broadband light coupling to high index substrates Sci. Rep. 638682

[41] Ji S, Song K, Nguyen T B, Kim N and Lim H 2013 Optimal moth eye nanostructure array on transparent glass towards broadband antireflection ACS Appl. Mater. Interfaces 5 10731-7

[42] Liu V and Fan S 2012 S4: a free electromagnetic solver for layered periodic structures Comput. Phys. Commun. 183 2233-44

[43] Boidin R, Halenkovič T, Nazabal V, Beneš L and Němec P 2016 Pulsed laser deposited alumina thin films Ceram. Int. 42 1177-82

[44] Tanemura S, Miao L, Jin P, Kaneko K, Terai A and Nabatova-Gabain N 2003 Optical properties of polycrystalline and epitaxial anatase and rutile $\mathrm{TiO}_{2}$ thin films by RF magnetron sputtering Appl. Surf. Sci. 212-213 654-60

[45] Palik E D (ed) 1998 Handbook of Optical Constants of Solids vol 3 (San Diego: Academic)

[46] Kato H, Adachi S, Nakanishi H and Ohtsuka K 1994 Optica properties of $\left(\mathrm{Al}_{x} \mathrm{Ga}_{1-x}\right)_{0.5} \mathrm{In}_{0.5} \mathrm{P}$ quaternary alloys Japan. J. Appl. Phys. 33 186-92

[47] Schubert M, Gottschalch V, Herzinger C M, Yao H, Snyder P G and Woollam J A 1995 Optical constants of $\mathrm{Ga}_{x} \operatorname{In}_{1-x} \mathrm{P}$ lattice matched to GaAs J. Appl. Phys. 77 3416-9

[48] Ferrini R, Guizzetti G, Patrini M, Parisini A, Tarricone L and Valenti B 2002 Optical functions of InGaP/GaAs epitaxial layers from 0.01 to $5.5 \mathrm{eV}$ Eur. Phys. J. B 27 449-58

[49] Nunley T N, Fernando N S, Samarasingha N, Moya J M, Nelson C M, Medina A A and Zollner S 2016 Optical constants of germanium and thermally grown germanium dioxide from 0.5 to $6.6 \mathrm{eV}$ via a multisample ellipsometry investigation J. Vac. Sci. Technol. B 34061205

[50] Geisz J F, Steiner M A, Garcia I, France R M, McMahon W E, Osterwald C R and Friedman D J 2015 Generalized optoelectronic model of series-connected multijunction solar cells IEEE J. Photovolt. 5 1827-39

[51] Ripalda J M, Buencuerpo J and García I 2018 Solar cell designs by maximizing energy production based on machine learning clustering of spectral variations $\mathrm{Nat}$. Commun. 95126

[52] Baur C, Gervasi M, Nieminen P, Pensotti S, Rancoita P g and Tacconi M 2014 NIEL dose dependence for solar cells irradiated with electrons and protons Astroparticle, Particle, Space Physics, Radiation Interaction, Detectors and Medical Physics Applications vol 8 (Singapore: World Scientific) pp 692-707

[53] Cappelletti M A, Cédola A P and Peltzer y Blancá E L 2014 Computational analysis of the maximum power point for GaAs sub-cells in InGaP/GaAs/Ge triple-junction space solar cells Semicond. Sci. Technol. 29115025

[54] Campesato R et al 2018 Effects of irradiation on triple and single junction InGaP/GaAs/Ge solar cells 35th European Photovoltaic Solar Energy Conf. and Exhibition (WIP) pp 959-64

[55] Leroy C and Rancoita P-G 2009 Principles of Radiation Interaction in Matter and Detection 2nd edn (Singapore: World Scientific)

[56] Boschini M J, Consolandi C, Gervasi M, Giani S, Grandi D, Ivanchenko V, Pensotti S, Rancoita P G and Tacconi M 2011 Nuclear and non-ionizing energy-loss for Coulomb scattered particles from low energy up to relativistic regime in space radiation environment Cosmic Rays for Particle and Astroparticle Physics (Singapore: World Scientific) pp 9-23

[57] Codegoni D et al 2004 Investigation of irradiated monolithic transistors for space applications Nucl. Instrum. Methods Phys. Res. B 217 65-76

[58] Messenger S R, Burke E A, Xapsos M A, Summers G P, Walters R J, Insoo Jun J and Jordan T 2003 NIEL for heavy ions: an analytical approach IEEE Trans. Nucl. Sci. 50 1919-23

[59] Jun I, Kim W and Evans R 2009 Electron nonionizing energy loss for device applications IEEE Trans. Nucl. Sci. 56 3229-35

[60] Insoo Jun I, Xapsos M A, Messenger S R, Burke E A, Walters R J, Summers G P and Jordan T 2003 Proton nonionizing energy loss (NIEL) for device applications IEEE Trans. Nucl. Sci. 50 1924-8

[61] Agostinelli S et al 2003 Geant4-a simulation toolkit Nucl. Instrum. Methods Phys. Res. A 506 250-303

[62] Vasileiou T and Summerer L 2020 A biomimetic approach to shielding from ionizing radiation: the case of melanized fungi PLoS One 15 e0229921

[63] Gruginskie N, Cappelluti F, Bauhuis G J, Mulder P, Haverkamp E J, Vlieg E and Schermer J J 2020 Electron radiation-induced degradation of GaAs solar cells with different architectures Prog. Photovolt. Res. Appl. 28 266-78

[64] Biscani F and Izzo D 2020 A parallel global multiobjective framework for optimization: pagmo J. Open Source Software 52338

[65] Messenger S R, Lorentzen J, Walters R J, Warner J H and Summers G P 2006 Electron to proton damage correlation in space solar cells 2006 IEEE 4th World Conf. on Photovoltaic Energy Conf. vol 2 pp 1781-4

[66] Bläsi B, Tucher N, Höhn O, Kübler V, Kroyer T, Wellens C and Hauser H 2016 Large area patterning using interference and nanoimprint lithography Micro-Optics 2016 vol 9888 ed $\mathrm{H}$ Thienpont, J Mohr, H Zappe and H Nakajima (Bellingham, WA: SPIE Optical Engineering Press) pp 80-8

[67] Rahman A, Ashraf A, Xin H, Tong X, Sutter P, Eisaman M D and Black C T 2015 Sub-50 nm self-assembled nanotextures for enhanced broadband antireflection in silicon solar cells Nat. Commun. 65963

[68] Leem J W, Su Yu J, Jun D-H, Heo J and Park W-K 2014 Efficiency improvement of III-V GaAs solar cells using biomimetic $\mathrm{TiO}_{2}$ subwavelength structures with wide-angle and broadband antireflection properties Sol. Energy Mater. Sol. Cells 127 43-9

[69] Spinelli P, Macco B, Verschuuren M A, Kessels W M M and Polman A $2013 \mathrm{Al}_{2} \mathrm{O}_{3} / \mathrm{TiO}_{2}$ nano-pattern antireflection coating with ultralow surface recombination Appl. Phys. Lett. 102233902 
[70] Parker G J, Charlton M D B, Zoorob M E, Baumberg J J, Netti M C and Lee T 2005 Highly engineered mesoporous structures for optical processing Phil. Trans. R. Soc. A 364 189-99

[71] Zaman S, Hassan M M, Hasanuzzaman M, Baten M Z, Hassan M M, Hasanuzzaman M and Zunaid Baten M 2020 Coscinodiscus diatom inspired bi-layered photonic structures with near-perfect absorptance accompanied by tunable absorption characteristics Opt. Express 28 25007-21

[72] Mellor A, Hylton N P, Maier S A and Ekins-Daukes N 2017 Interstitial light-trapping design for multi-junction solar cells Sol. Energy Mater. Sol. Cells 159 212-8

[73] Messenger S R, Burke E A, Walters R J, Warner J H, Summers G P and Morton T L 2006 Effect of omnidirectional proton irradiation on shielded solar cells IEEE Trans. Nucl. Sci. 53 3771-8

[74] Messenger S R, Wong F, Hoang B, Cress C D, Walters R J, Kluever C A and Jones G 2014 Low-thrust geostationary transfer orbit (LT2GEO) radiation environment and associated solar array degradation modeling and ground testing IEEE Trans. Nucl. Sci. 61 3348-55
[75] Fonash S J 2010 Solar Cell Device Physics 2nd edn (Burlington, MA: Academic)

[76] Sotoodeh M, Khalid A H and Rezazadeh A A 2000 Empirical low-field mobility model for III-V compounds applicable in device simulation codes J. Appl. Phys. 87 2890-900

77] Ivantchenko A V, Ivanchenko V N, Quesada Molina J-M and Incerti S L 2012 Geant4 hadronic physics for space radiation environment Int. J. Radiat. Biol. 88 171-5

[78] Boschini M J et al 2012 Nuclear and non-ionizing energy-loss of electrons with low and relativistic energies in materials and space environment Astroparticle, Particle, Space Physics and Detectors for Physics Applications (Singapore: World Scientific) pp 961-82

[79] Ivanchenko V N, Kadri O, Maire M and Urban L 2010 Geant 4 models for simulation of multiple scattering J. Phys.: Conf. Ser. 219032045

[80] Heynderickx D, Quaghebeur B, Wera J, Daly E J and Evans H D R 2004 New radiation environment and effects models in the European space agency's space environment information system (SPENVIS) Space Weather 2 $\mathrm{S} 10 \mathrm{~S} 03$ 